\title{
Based on Multi-Activity Integrated Strategy to Screening, Characterization and Quantification of Bioactive Compounds from Red Wine
}

\author{
Yuye Gao ${ }^{1,+}$, Xie-an Yu ${ }^{2,+} \mathbb{D}$, Bing Wang ${ }^{2}$, Guo Yin ${ }^{2}$, Jue Wang ${ }^{2}$, Tiejie Wang ${ }^{2, *}$ and Kaishun Bi ${ }^{1, *}$ \\ 1 School of Pharmacy, Shenyang Pharmaceutical University, Shenyang 110016, China; 13002480316@163.com \\ 2 NMPA Key Laboratory for Quality Research and Evaluation of Traditional Chinese Medicine, \\ Shenzhen Institute for Drug Control, Shenzhen 518057, China; yuxieanalj@126.com (X.-a.Y.); \\ wangbing@szidc.org.cn (B.W.); ayinguoa@126.com (G.Y.); wangjuepha@126.com (J.W.) \\ * Correspondence: szyjwtj@163.com (T.W.); kaishunbi.syphu@gmail.com (K.B.) \\ + These authors contributed equally to this work.
}

check for updates

Citation: Gao, Y.; Yu, X.-a.; Wang, B.; Yin, G.; Wang, J.; Wang, T.; Bi, K. Based on Multi-Activity Integrated Strategy to Screening,

Characterization and Quantification of Bioactive Compounds from Red Wine. Molecules 2021, 26, 6750. https: / / doi.org/10.3390/

molecules26216750

Academic Editor: Petr Bednar

Received: 3 September 2021

Accepted: 5 November 2021

Published: 8 November 2021

Publisher's Note: MDPI stays neutral with regard to jurisdictional claims in published maps and institutional affiliations.

Copyright: (c) 2021 by the authors. Licensee MDPI, Basel, Switzerland. This article is an open access article distributed under the terms and conditions of the Creative Commons Attribution (CC BY) license (https:/ / creativecommons.org/licenses/by/ $4.0 /)$.

\begin{abstract}
According to French Paradox, red wine was famous for the potential effects on coronary heart disease (CHD), but the specific compounds against CHD were unclear. Therefore, screening and characterization of bioactive compounds from red wine was extremely necessary. In this paper, the multi-activity integrated strategy was developed and validated to screen, identify and quantify active compounds from red wine by using ultra high performance liquid chromatographyfraction collector (UHPLC-FC), ultra fast liquid chromatography-quadrupole-time-of-flight/mass spectrometry (UFLC-Q-TOF/MS) and bioactive analysis. UHPLC-FC was employed to separate and collect the components from red wine, which was further identified by UFLC-Q-TOF/MS to acquire their structural information. Furthermore, the active fractions were tested for antioxidant activity, inhibitory activity against thrombin and lipase activities in vitro by the activity screening kit. As the results, there were 37 fractions had antioxidant activity, 22 fractions had thrombin inhibitory activity and 28 fractions had lipase inhibitory activity. Finally, 77 active components from red wine were screened and 12 ingredients out of them were selected for quantification based on the integration of multi-activity. Collectively, the multi-activity integrated strategy was helpful for the rapid and effective discovery of bioactive components, which provided reference for exploring the health care function of food.
\end{abstract}

Keywords: red wine; multi-activity integrated strategy; active components; coronary heart disease

\section{Introduction}

As a nutritious drink recommended by World Health Organization (WHO), red wine was widely recognized for its role in health care. According to Ben Cao Gang Mu (Compendium of Materia Medica) record, the famous classic book of traditional Chinese medicine, red wine could treat cold coagulation and blood stasis. Modern pharmacological studies showed that red wine had the function of antioxidant, anticoagulant and lipidlowering [1,2], which can prevent cardiovascular and cerebrovascular diseases such as coronary heart disease (CHD) [3,4].

It was widely believed that the mechanisms of CHD were the result of the interaction of many complex factors, among them the "response to injury hypothesis" had been recognized by researchers [5,6], which stated that the initial damage was the arterial endothelium, further leading to endothelial dysfunction [7]. Indeed, the occurrence of CHD was caused by many factors such as oxidative stress response, hyperlipidemia and platelet aggregation [8]. There were three main methods to treat CHD including pharmacotherapy, interventional therapy and operation $[9,10]$. Although these methods hold the potential effects for CHD, these methods would also lead some side effects 
such as common complications after coronary intervention included bleeding, pulmonary embolism, infection and so on. Besides, long term use of some medicines as nitroglycerin would also generate drug resistance and migraine pain [11]. Therefore, prevention CHD has become the trend of medical development.

The famous French paradox proved that red wine can prevent CHD [3]. Accumulated studies demonstrated that extracts from red wine possessed antioxidant activity, anticoagulation and lipid-lowering efficacy, which corresponded to the pathogenesis of CHD. Traditional methods for isolating and evaluating components were most carried out on animal and cell models, which were time-consuming, labor-intensive, expensive and hardly to be used for the direct screening [12]. The developed methods for screening active compounds were extracted, the single component from sample and evaluated the biological activity by pharmacological means. Animal model was the most important screening manner in the part of bioactive components evaluation, which can clearly response to the efficacy of ingredients in the whole body. However, the method was high cost and low efficiency. Besides, compared with the animal model, cellular model was relatively cheap and convenient, but it had higher requirement on instrumentation [13]. Therefore, it was urgent to establish a fast, portable and accurate method to screen and identify the bioactive ingredients from red wine. Ultra high performance liquid chromatography (UHPLC) was used to separate the components of food with appropriate chromatographic conditions and the fraction collector was used to enrich compounds automatically in the fixed-time interval according to the retention time in the UHPLC chromatogram. Compared with HPLC, UHPLC exhibited higher separation and narrower chromatographic peak. At the same time, ACQUITY UPLC column (HSS T3, $1.8 \mu \mathrm{m}, 2.1 \mathrm{~mm} \times 100 \mathrm{~mm}$, Waters, Ireland) could withstand $100 \%$ water mobile phase and the particle size was only 1.8 microns, which was adapted to separation of complex samples especially for the strong polar compounds from red wine. Indeed, some researches showed that ultra high performance liquid chromatography-fraction collector (UHPLC-FC) could quickly separate and enrich compounds. Moreover, ultra fast liquid chromatography-quadrupole-timeof-flight/mass spectrometry (UFLC-Q-TOF/MS) was used to identify the components for obtaining structural information [12]. Accordingly, integrated the UHPLC-FC and UFLC-Q-TOF/MS could be used for quickly separating, enriching and identifying the potential active compounds from complex samples.

In this study, UHPLC-FC was used to separate, collect and enrich the fractions from red wine. Then antioxidant activity, inhibitory activity against thrombin and lipase activities of all fractions were performed for evaluating activities. Furthermore, UFLC-Q-TOF/MS was used to identify the components in fractions from red wine (Figure 1). As the results, 77 potential active components from red wine were screened and 12 ingredients out of them were selected for quantification based on integration of the multi-activity. Accordingly, the multi-activity integrated strategy was significant for the rapid and effective discovery of the potential bioactive components from red wine to prevent CHD, which provided reference for exploring the health care function of food.

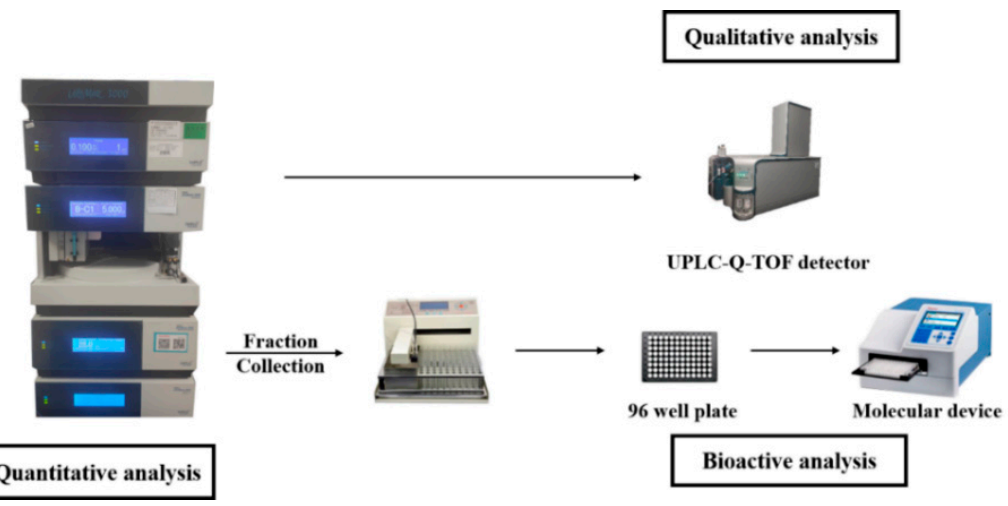

Figure 1. Schematic diagram of principle for screening active ingredient. 


\section{Result and Discussion}

\subsection{Establishment of Multi-Activity Integrated Strategy}

In the study, an ultra high performance liquid chromatography-photo-diode arrayfraction collector (UHPLC-PDA-FC) system was used to establish multi-activity integrated strategy for screening and evaluating the potentially bioactive components from red wine. The sample was divided into sixty fractions (60 s per fraction) by UHPLC-PDA-FC. Sixty fractions were used to screen the antioxidant activity, thrombin inhibitory activity and lipase inhibitory activity. The multi-activity integrated chromatogram consisted of chromatogram and multi-targets integrated column diagram of antioxidant activity, inhibitory activity of thrombin and lipase were established (Figure 2) and the detail data was listed in Table 1. The multi-activity integrated strategy could discover bioactive components rapidly and efficiently, which could be used for the identification and screening the active compounds from nutraceutical. According to the activities results, the activities were higher at 20 to $30 \mathrm{~min}$, indicating that these kinds of ingredients may be the main active compounds from red wine.

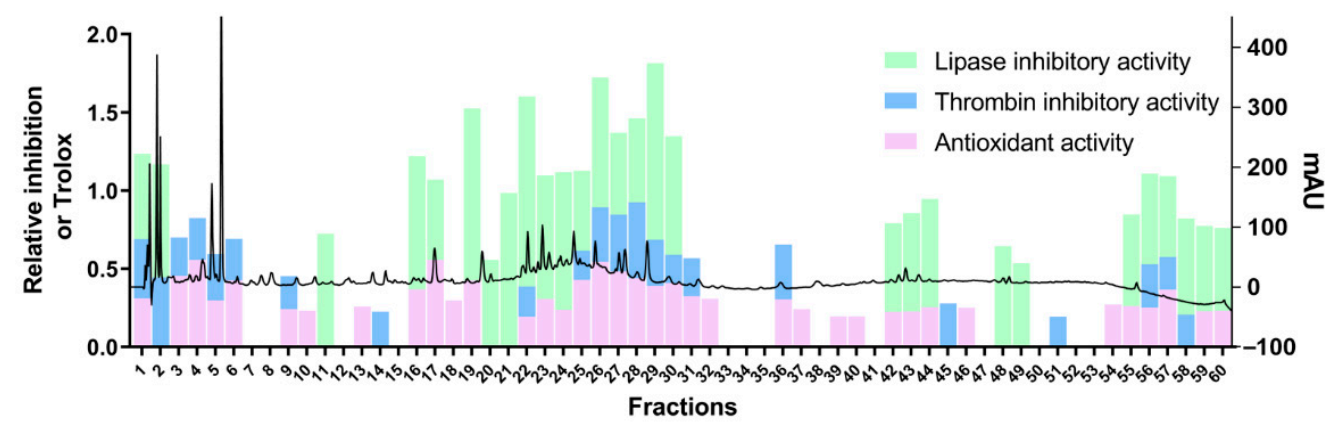

Figure 2. The multi-activity integrated chromatogram of antioxidant activity, inhibitory activity of thrombin and inhibitory activity of lipase. The chromatogram of red wine was shown in superstratum, substratum was a column diagram of each fraction for the three activities.

Table 1. The data of antioxidant activity (expressed in Trolox), thrombin inhibitory activity (expressed in inhibition) and lipase inhibitory activity (expressed in inhibition).

\begin{tabular}{cccc}
\hline & $\begin{array}{c}\text { Antioxidant Activity } \\
\text { Inhibition (Trolox (\%)) }\end{array}$ & $\begin{array}{c}\text { Thrombin Inhibitory } \\
\text { Activity Inhibition (\%) }\end{array}$ & $\begin{array}{c}\text { Lipase Inhibitory } \\
\text { Activity Inhibition (\%) }\end{array}$ \\
\hline F1 & 31.07 & 37.82 & 69.67 \\
F2 & no & 44.42 & 87.30 \\
F3 & 45.35 & 24.65 & no \\
F4 & 55.64 & 26.68 & no \\
F5 & 29.63 & 29.77 & no \\
F6 & 42.56 & 26.61 & no \\
F7 & no & no & no \\
F8 & no & no & no \\
F9 & 24.08 & 21.21 & no \\
F10 & 23.10 & no & no \\
F11 & no & no & 87.35 \\
F12 & no & no & no \\
F13 & 25.70 & no & no \\
F14 & no & 22.44 & no \\
F15 & no & no & no \\
\hline
\end{tabular}


Table 1. Cont.

\begin{tabular}{|c|c|c|c|}
\hline & $\begin{array}{c}\text { Antioxidant Activity } \\
\text { Inhibition (Trolox (\%)) }\end{array}$ & $\begin{array}{l}\text { Thrombin Inhibitory } \\
\text { Activity Inhibition (\%) }\end{array}$ & $\begin{array}{c}\text { Lipase Inhibitory } \\
\text { Activity Inhibition (\%) }\end{array}$ \\
\hline F16 & 36.79 & no & 100.20 \\
\hline F17 & 55.64 & no & 66.44 \\
\hline F18 & 29.63 & no & no \\
\hline F19 & 42.56 & no & 124.96 \\
\hline F20 & no & no & 70.68 \\
\hline F21 & no & no & 113.43 \\
\hline F22 & 19.34 & 19.22 & 136.53 \\
\hline F23 & 30.56 & no & 94.14 \\
\hline F24 & 23.59 & no & 103.15 \\
\hline F25 & 42.87 & 18.56 & 66.12 \\
\hline F26 & 54.32 & 35.06 & 97.89 \\
\hline F27 & 48.31 & 36.21 & 67.39 \\
\hline F28 & 43.88 & 48.47 & 68.79 \\
\hline F29 & 39.03 & 29.44 & 127.97 \\
\hline F30 & 40.83 & 18.07 & 90.74 \\
\hline F31 & 32.31 & 24.49 & no \\
\hline F32 & 30.82 & no & no \\
\hline F33 & no & no & no \\
\hline F34 & no & no & no \\
\hline F35 & no & no & no \\
\hline F36 & 30.36 & 35.12 & no \\
\hline F37 & 24.07 & no & no \\
\hline F38 & no & no & no \\
\hline F39 & 19.44 & no & no \\
\hline F40 & 19.48 & no & no \\
\hline F41 & no & no & no \\
\hline F42 & 22.28 & no & 71.92 \\
\hline F43 & 22.64 & no & 77.88 \\
\hline S44 & 25.36 & no & 84.26 \\
\hline F45 & no & 27.81 & no \\
\hline F46 & 25.08 & no & no \\
\hline F47 & no & no & no \\
\hline F48 & no & no & 79.38 \\
\hline F49 & no & no & 68.49 \\
\hline F50 & no & no & no \\
\hline F51 & no & 19.33 & no \\
\hline F52 & no & no & no \\
\hline F53 & no & no & no \\
\hline F54 & 27.09 & no & no \\
\hline F55 & 25.94 & no & 73.85 \\
\hline F56 & 25.27 & 27.66 & 72.88 \\
\hline F57 & 36.68 & 20.91 & 66.70 \\
\hline F58 & no & 20.65 & 76.42 \\
\hline F59 & 22.80 & no & 69.65 \\
\hline F60 & 23.21 & no & 67.84 \\
\hline
\end{tabular}

\subsection{Identification of 94 Components from Red Wine}

The components from red wine with antioxidant activity or inhibitory potential against thrombin and lipase activities were analyzed by UFLC-Q-TOF/MS to obtain structural information. The molecular compositions of ingredients were identified according to the literature and exact molecular weight. The peak number, retention time, MS/MS fragmentation ions information and identification of 94 components from red wine were listed in Table 2. The extracted ion chromatogram of positive and negative ions was illustrated in Figure 3. Peak 1 gave the mass spectrum ion at $116.0702\left(\mathrm{C}_{5} \mathrm{H}_{9} \mathrm{NO}_{2}\right)$ with $\mathrm{MS} / \mathrm{MS}$ fragment of $70.0647\left(\mathrm{C}_{4} \mathrm{H}_{7} \mathrm{~N}\right)[\mathrm{M}+\mathrm{H}-\mathrm{HCOOH}]^{+}$, which was detected to be D-proline [14-16]. Peaks $11\left(\mathrm{C}_{4} \mathrm{H}_{9} \mathrm{~N}\right)[\mathrm{M}+\mathrm{H}-\mathrm{HCOOH}]^{+}$and $14\left(\mathrm{C}_{5} \mathrm{H}_{3} \mathrm{~N}\right)[\mathrm{M}+\mathrm{H}-\mathrm{HCOOH}]^{+}$were based on 
the same regular. Therefore, peaks 11 and 14 were identified as L-valine $\left(\mathrm{C}_{5} \mathrm{H}_{11} \mathrm{NO}_{2}\right)$ [14] and niacin $\left(\mathrm{C}_{6} \mathrm{H}_{5} \mathrm{NO}_{2}\right)$ [17]. Peak 2 had the mass spectrum ion at $175.1189\left(\mathrm{C}_{6} \mathrm{H}_{14} \mathrm{~N}_{4} \mathrm{O}_{2}\right)$ with MS/MS fragment of $70.0652\left[\mathrm{M}+\mathrm{H}-\mathrm{HCOOH}-2 \mathrm{NH}_{2}-\mathrm{CN}\right]^{+}$, which was detected to be L-arginine [14]. Peak 3 had a molecular weight of $179.0562\left(\mathrm{C}_{6} \mathrm{H}_{12} \mathrm{O}_{6}\right)$ with fragments of $99.0087\left(\mathrm{C}_{4} \mathrm{H}_{4} \mathrm{O}_{3}\right)\left[\mathrm{M}-\mathrm{H}-3 \mathrm{H}_{2} \mathrm{O}_{-} \mathrm{C}_{2} \mathrm{HO}\right]^{-}, 71.0137\left(\mathrm{C}_{3} \mathrm{H}_{4} \mathrm{O}_{2}\right)\left[\mathrm{M}-\mathrm{H}-3 \mathrm{H}_{2} \mathrm{O}-\mathrm{C}_{2} \mathrm{HO}-\mathrm{CO}\right]^{-}$ and $59.0139\left(\mathrm{C}_{2} \mathrm{H}_{4} \mathrm{O}_{2}\right)\left[\mathrm{M}-\mathrm{H}-3 \mathrm{H}_{2} \mathrm{O}-\mathrm{C}_{2} \mathrm{HO}-\mathrm{CO}-\mathrm{CH}_{2}\right]^{-}$. Therefore, it was identified as Dglucose. Peak 4 had mass spectrum ion at $181.072\left(\mathrm{C}_{6} \mathrm{H}_{14} \mathrm{O}_{6}\right)$ with MS/MS experiment gave fragmentation information for $83.0143\left[\mathrm{M}-\mathrm{H}-\mathrm{C}_{3} \mathrm{H}_{2} \mathrm{O}_{3}\right]^{-}$and $71.0140\left[\mathrm{M}-\mathrm{H}_{-}-\mathrm{C}_{3} \mathrm{H}_{6} \mathrm{O}_{3}-\right.$ $\mathrm{H}_{2} \mathrm{O}^{-}$, which could be identified as D-mannitol [18]. Peak 5 had major ions at $\mathrm{m} / \mathrm{z}$ $191.0199\left(\mathrm{C}_{6} \mathrm{H}_{8} \mathrm{O}_{7}\right)$ and the MS/MS fragments were $85.0296\left(\mathrm{C}_{4} \mathrm{H}_{6} \mathrm{O}_{2}\right)\left[\mathrm{M}-\mathrm{H}-2 \mathrm{CO}_{2}-\mathrm{H}_{2} \mathrm{O}\right]^{-}$ and $59.0134\left(\mathrm{C}_{3} \mathrm{H}_{8} \mathrm{O}\right)\left[\mathrm{M}-\mathrm{H}-3 \mathrm{CO}_{2}\right]^{-}$. Therefore, it was identified as citric acid [19]. Peak 6 was identified as tartaric acid by its characteristic fragmentation pattern, which MS/MS spectra ions m/z $105.0217\left(\mathrm{C}_{3} \mathrm{H}_{6} \mathrm{O}_{4}\right)\left[\mathrm{M}-\mathrm{H}-\mathrm{CO}_{2}\right]^{-}$and $87.0085\left(\mathrm{C}_{3} \mathrm{H}_{4} \mathrm{O}_{3}\right)\left[\mathrm{M}-\mathrm{H}-\mathrm{CO}_{2}-\mathrm{H}_{2} \mathrm{O}\right]^{-}$ were observed [20]. By the similar regular pattern, peak $40\left(\mathrm{C}_{9} \mathrm{H}_{8} \mathrm{O}_{3}\right)$ had the MS/MS fragment of $\mathrm{m} / \mathrm{z} 119.0502\left(\mathrm{C}_{8} \mathrm{H}_{8} \mathrm{O}\right)\left[\mathrm{M}-\mathrm{H}-\mathrm{CO}_{2}\right]^{-}$. Therefore, Peak 40 was identified as 2-hydroxycinnamic acid [21]. Peak 7, the molecular weight was $138.0548\left(\mathrm{C}_{7} \mathrm{H}_{7} \mathrm{NO}_{2}\right)$ and the fragment ions observed included $\mathrm{m} / \mathrm{z} 120.0433\left(\mathrm{C}_{6} \mathrm{H}_{7} \mathrm{~N}\right)\left[\mathrm{M}+\mathrm{H}-\mathrm{H}_{2} \mathrm{O}\right]^{+}, 78.0339\left(\mathrm{C}_{6} \mathrm{H}_{6}\right)$ $\left[\mathrm{M}+\mathrm{H}-\mathrm{HCOOH}-\mathrm{NH}_{2}\right]^{+}$, which was identified to be 4 -aminobenzoic acid [22]. Peak 8 gave a mass spectrum ion at $341.1088\left(\mathrm{C}_{12} \mathrm{H}_{22} \mathrm{O}_{11}\right)$ with MS/MS fragments of $161.0459\left(\mathrm{C}_{6} \mathrm{H}_{9} \mathrm{O}_{5}\right)$ [M-H- $\left.\mathrm{C}_{6} \mathrm{H}_{11} \mathrm{O}_{5}-\mathrm{H}_{2} \mathrm{O}\right]^{-}$and $71.0137\left(\mathrm{C}_{3} \mathrm{H}_{4} \mathrm{O}_{2}\right)\left[\mathrm{M}-\mathrm{H}-\mathrm{C}_{9} \mathrm{H}_{14} \mathrm{O}_{8}-\mathrm{H}_{2} \mathrm{O}\right]^{-}$, which was detected to be trehalose [23]. The molecular weight of peak 9 was $149.0455\left(\mathrm{C}_{5} \mathrm{H}_{10} \mathrm{O}_{5}\right)$. In the MS/MS experiment, the predominant ions appeared at $75.0221\left(\mathrm{C}_{3} \mathrm{H}_{7} \mathrm{O}_{2}\right)\left[\mathrm{M}-\mathrm{H}-\mathrm{C}_{2} \mathrm{H}_{3} \mathrm{O}_{3}\right]^{-}$ and $59.0133\left(\mathrm{C}_{2} \mathrm{H}_{2} \mathrm{O}_{2}\right)\left[\mathrm{M}-\mathrm{H}-\mathrm{C}_{3} \mathrm{H}_{8} \mathrm{O}_{2}\right]^{-}$. By comparing the characteristic fragmentation pattern with the reported in references, it can be identified as D-ribose. Peaks $10\left(\mathrm{C}_{4} \mathrm{H}_{6} \mathrm{O}_{5}\right)$ was identified as L-malic acid [24] which the $\mathrm{H}_{2} \mathrm{O}$ was removed at first, and then the $\mathrm{CO}_{2}$ was removed. MS/MS fragment of L-malic acid was $115.0018\left(\mathrm{C}_{4} \mathrm{H}_{4} \mathrm{O}_{4}\right)\left[\mathrm{M}-\mathrm{H}-\mathrm{H}_{2} \mathrm{O}\right]^{-}$ and $71.0137\left(\mathrm{C}_{3} \mathrm{H}_{4} \mathrm{O}_{2}\right)\left[\mathrm{M}-\mathrm{H}-\mathrm{H}_{2} \mathrm{O}-\mathrm{CO}_{2}\right]^{-}$. Peaks $12\left(\mathrm{C}_{7} \mathrm{H}_{10} \mathrm{O}_{5}\right)$ was identified as shikimic acid. MS/MS fragment was $137.0225\left(\mathrm{C}_{7} \mathrm{H}_{6} \mathrm{O}_{3}\right)\left[\mathrm{M}-\mathrm{H}-2 \mathrm{H}_{2} \mathrm{O}\right]^{-}$. The molecular weight of peak 13 was $150.0586\left(\mathrm{C}_{5} \mathrm{H}_{11} \mathrm{NO}_{2} \mathrm{~S}\right)$ and ion $\mathrm{m} / \mathrm{z} 74.0243\left(\mathrm{C}_{3} \mathrm{H}_{8} \mathrm{O}_{2}\right)\left[\mathrm{M}+\mathrm{H}-\mathrm{NH}_{3}-\mathrm{C}_{2} \mathrm{~S}\right]^{+}$was observed in MS/MS spectra, which could be identified as L-methionine [16]. Peak 15 had a molecular weight of $117.0192\left(\mathrm{C}_{4} \mathrm{H}_{6} \mathrm{O}_{4}\right)$, which produced the MS/MS ions as 99.9254 $\left(\mathrm{C}_{3} \mathrm{H}_{6} \mathrm{O}_{2}\right)\left[\mathrm{M}-\mathrm{H}-\mathrm{CO}_{2}\right]^{-}$and $73.0291\left(\mathrm{C}_{3} \mathrm{H}_{4} \mathrm{O}\right)\left[\mathrm{M}-\mathrm{H}-\mathrm{CO}_{2}-\mathrm{H}_{2} \mathrm{O}\right]^{-}$. Hence, it can be identified as succinic acid [25]. Peak 33 had the same fragment $\left(73.0273\left[\mathrm{M}-\mathrm{H}_{-}-\mathrm{C}_{3} \mathrm{H}_{5} \mathrm{O}_{2}\right]^{-}\right)$with peak 15. The other fragment was $55.0191\left[\mathrm{M}-\mathrm{H}_{-}-\mathrm{C}_{3} \mathrm{H}_{5} \mathrm{O}_{2}-\mathrm{H}_{2} \mathrm{O}\right]^{-}$, which can be identified as dimethyl succinate [25]. Peaks 18, 21, 23, 24, 25, 29, 31, 32, 33, 39, 42, 43, 49, 50, 52, 55, 56, 60 and 61 were in the similar regular pattern with peak 15 , the parent ion was removed one or more $\mathrm{CO}_{2}$ and $\mathrm{H}_{2} \mathrm{O}$. Peaks $18\left(125.0244\left[\mathrm{M}-\mathrm{H}-\mathrm{CO}_{2}\right]^{-}, 107.0156\left[\mathrm{M}-\mathrm{H}-\mathrm{CO}_{2}-\mathrm{H}_{2} \mathrm{O}\right]^{-}\right.$ and $\left.79.0189\left[\mathrm{M}-\mathrm{H}-\mathrm{CO}_{2}-\mathrm{H}_{2} \mathrm{O}-\mathrm{CO}\right]^{-}\right), 21\left(109.0298\left[\mathrm{M}-\mathrm{H}-\mathrm{CO}_{2}\right]^{-}\right.$and $91.0298\left[\mathrm{M}-\mathrm{H}_{-}-\mathrm{CO}_{2}{ }^{-}\right.$ $\left.\left.\mathrm{H}_{2} \mathrm{O}\right]^{-}\right), 23\left(135.0560\left[\mathrm{M}-\mathrm{H}-\mathrm{CO}_{2}\right]^{-}\right.$and $\left.89.0194\left[\mathrm{M}-\mathrm{H}-\mathrm{CO}_{2}-\mathrm{CH}_{3} \mathrm{CO}\right]^{-}\right), 24(135.0454[\mathrm{M}-\mathrm{H}-$ $\left.\mathrm{CO}_{2}\right]^{-}$and $\left.117.0351\left[\mathrm{M}-\mathrm{H}-\mathrm{CO}_{2}-\mathrm{H}_{2} \mathrm{O}\right]^{-}\right), 25\left(109.0298\left[\mathrm{M}-\mathrm{H}_{-} \mathrm{CO}_{2}\right]^{-}\right.$and $91.0298\left[\mathrm{M}-\mathrm{H}-\mathrm{CO}_{2}-\right.$ $\left.\left.\mathrm{H}_{2} \mathrm{O}\right]^{-}\right), 29\left(93.0340\left[\mathrm{M}-\mathrm{H}-\mathrm{CO}_{2}\right]^{-}\right), 31\left(163.0400\left[\mathrm{M}-\mathrm{H}-2 \mathrm{CO}_{2}\right]^{-}\right.$and $\left.119.0503\left[\mathrm{M}-\mathrm{H}-3 \mathrm{CO}_{2}\right]^{-}\right)$, $32\left(119.0498\left[\mathrm{M}-\mathrm{H}-\mathrm{CO}_{2}\right]^{-}\right.$and $\left.93.0347\left[\mathrm{M}-\mathrm{H}-\mathrm{CO}_{2}-\mathrm{H}_{2} \mathrm{O}\right]^{-}\right), 33\left(163.0400\left[\mathrm{M}-\mathrm{H}-2 \mathrm{CO}_{2}\right]^{-}\right.$ and $\left.119.0498\left[\mathrm{M}-\mathrm{H}-3 \mathrm{CO}_{2}\right]^{-}\right), 39\left(108.0212\left[\mathrm{M}-\mathrm{H}-\mathrm{CO}_{2}-\mathrm{CH}_{3}\right]^{-}\right), 42\left(193.0490\left[\mathrm{M}-\mathrm{H}-2 \mathrm{CO}_{2}-\right.\right.$ $\left.\mathrm{C}_{2} \mathrm{H}_{5} \mathrm{O}\right]^{-}$and $\left.117.0341\left[\mathrm{M}-\mathrm{H}-3 \mathrm{CO}_{2}-\mathrm{C}_{2} \mathrm{H}_{5} \mathrm{O}-\mathrm{CH}_{3}-\mathrm{H}_{2} \mathrm{O}\right]^{-}\right), 43\left(135.0449\left[\mathrm{M}-\mathrm{H}-\mathrm{CO}_{2}\right]^{-}\right), 49$ $\left(119.0527\left[\mathrm{M}-\mathrm{H}-\mathrm{CO}_{2}\right]^{-}\right.$and $\left.103.0565\left[\mathrm{M}-\mathrm{H}-\mathrm{CO}_{2}-\mathrm{H}_{2} \mathrm{O}\right]^{-}\right), 50\left(93.0470\left[\mathrm{M}-\mathrm{H}-\mathrm{CO}_{2}\right]^{-}\right.$and $\left.65.0473\left[\mathrm{M}-\mathrm{H}-\mathrm{CO}_{2}-\mathrm{CO}\right]^{-}\right), 52\left(123.0436\left[\mathrm{M}-\mathrm{H}-\mathrm{CO}_{2}\right]^{-}\right.$and $\left.108.0212\left[\mathrm{M}-\mathrm{H}-\mathrm{CO}_{2}-\mathrm{H}_{2} \mathrm{O}\right]^{-}\right)$, $55\left(119.0503\left[\mathrm{M}-\mathrm{H}-\mathrm{CO}_{2}\right]^{-}\right.$and $\left.93.0347\left[\mathrm{M}-\mathrm{H}-\mathrm{CO}_{2}-\mathrm{H}_{2} \mathrm{O}\right]^{-}\right), 56\left(123.0450\left[\mathrm{M}-\mathrm{H}-\mathrm{CO}_{2}\right]^{-}\right)$, $60\left(257.0110\left[\mathrm{M}-\mathrm{H}-\mathrm{CO}_{2}\right]^{-}\right.$and $\left.229.0126\left[\mathrm{M}-\mathrm{H}-\mathrm{CO}_{2}-\mathrm{CO}\right]^{-}\right)$and $61\left(109.0286\left[\mathrm{M}-\mathrm{H}-\mathrm{CO}_{2}{ }^{-}\right.\right.$ $\left.\mathrm{C}_{2} \mathrm{H}_{2}\right]^{-}$) were the same regular, which were identified as gallic acid [20], protocatechuic acid [23], acetylsalicylic acid [26], caftaric acid [20], gentisic acid [23], 4-hydroxybenzoic acid [27], p-coutaric acid [28], m-coumaric acid [28], coutaric acid [28], vanillic acid [27], fertaric acid [28], caffeic acid [28], desaminotyrosine [29], 2-hydroxybenzoic acid [28], 3-hydroxy-4-methoxybenzoic acid [30,31], p-coumaric acid [28], homogentisic acid [23], ellagic acid [32] and dihydrocaffeic acid [33]. Peak 79 had the major first-order mass 
spectrum at $\mathrm{m} / \mathrm{z} 270.0662\left(\mathrm{C}_{11} \mathrm{H}_{12} \mathrm{O}_{4}\right)$. The MS/MS fragments were the same as peak 43 of $179.0433\left[\mathrm{M}-\mathrm{H}-\mathrm{CH}_{3}\right]^{-}$and $135.0446\left[\mathrm{M}-\mathrm{H}-\mathrm{CH}_{3}-\mathrm{CO}_{2}\right]^{-}$. Therefore, it was identified as ethyl caffeate [28]. Peak 16 showed the mass spectrum ion at $\mathrm{m} / \mathrm{z} 165.0547\left(\mathrm{C}_{9} \mathrm{H}_{8} \mathrm{O}_{3}\right)$ and the product ion was $119.0479[\mathrm{M}+\mathrm{H}-\mathrm{HCOOH}]^{+}$, which could be identified as trans-4hydroxycinnamic acid [20]. Peak 17 gave a mass spectrum ion at $138.0548\left(\mathrm{C}_{8} \mathrm{H}_{11} \mathrm{NO}\right)$ with the MS/MS fragments at $121.0616\left[\mathrm{M}+\mathrm{H}-\mathrm{NH}_{3}\right]^{+}$and $95.0561\left[\mathrm{M}+\mathrm{H}-\mathrm{NH}_{3}-\mathrm{C}_{2} \mathrm{H}_{4}\right]^{+}$, which was detected to be tyramine [34,35]. Peak 19 showed the mass spectrum ion at 141.0182 with the MS/MS fragments at $95.0127[\mathrm{M}+\mathrm{H}-\mathrm{HCOOH}]^{+}$, which could be identified as coumalic acid. Peak 20 gave $[\mathrm{M}-\mathrm{H}]^{-}$ion at $\mathrm{m} / \mathrm{z}$ 153.0558. In MS/MS mode, the produced ions were $\mathrm{m} / \mathrm{z} 123.0085\left[\mathrm{C}_{8} \mathrm{H}_{10} \mathrm{O}_{3}-\mathrm{CH}_{2} \mathrm{O}\right]^{-}$and $108.0221\left[\mathrm{C}_{8} \mathrm{H}_{10} \mathrm{O}_{3}-\mathrm{CH}_{2} \mathrm{OH}-\mathrm{CH}_{3}\right]^{-}$, which can be identified as hydroxytyrosol [36]. Peaks 22 and 37 were a type of isomer which yielded the same ion at $305.0246[\mathrm{M}-\mathrm{H}]^{-}$in the first-order mass spectrum. They had the same prominent ion at $125.0246\left[\mathrm{C}_{15} \mathrm{H}_{14} \mathrm{O}_{7}-\mathrm{C}_{9} \mathrm{H}_{8} \mathrm{O}_{4}\right]^{-}$. They were speculated to be (-) epigallocatechin [25] and (+)-gallocatechin [25] based on their retention times. Peak 26 had the $[\mathrm{M}-\mathrm{H}]^{-}$ion at $\mathrm{m} / \mathrm{z}$ 137.0255. The product ion was $108.0213[\mathrm{M}-\mathrm{H}-\mathrm{CHO}]^{-}$. Therefore, peak 26 was identified as protocatechualdehyde. Peak 27 had the mass spectrum ion at $181.0506\left(\mathrm{C}_{9} \mathrm{H}_{10} \mathrm{O}_{4}\right)$ with MS/MS fragment of $135.0453\left[\mathrm{M}-\mathrm{H}-\mathrm{CH}_{3}-\mathrm{H}_{2} \mathrm{O}\right]^{-}$, which was identified as homovanillic acid [34,37]. Peak 28 showed mass spectrum ion at $183.0299\left(\mathrm{C}_{8} \mathrm{H}_{8} \mathrm{O}_{5}\right)$. The product fragment ions were $124.0234\left[\mathrm{M}-\mathrm{H}-\mathrm{CO}_{2}-\mathrm{CH}_{3}\right]^{-}$and $95.0136\left[\mathrm{M}-\mathrm{H}-\mathrm{CO}_{2}-\mathrm{CH}_{3}-\right.$ $\mathrm{CHO}^{-}$. Hence, it was identified as methyl gallate $[38,39]$. Peak 30 gave $[\mathrm{M}-\mathrm{H}]^{-}$ion at $\mathrm{m} / \mathrm{z}$ 353.0877 and MS/MS produced ions were at $\mathrm{m} / \mathrm{z} 191.0616\left[\mathrm{M}-\mathrm{H}_{-}-\mathrm{C}_{6} \mathrm{H}_{5} \mathrm{O}_{5}\right]^{-}$and 135.0439 $\left[\mathrm{M}-\mathrm{H}-\mathrm{C}_{6} \mathrm{H}_{5} \mathrm{O}_{5}-\mathrm{CO}\right]^{-}$, it can be identified as chlorogenic acid $[27,40]$. Peak 35 was identified as benzoic acid by the MS spectra ion 121.0296 and MS/MS spectra ions $\mathrm{m} / \mathrm{z} 92.0265$ $[\mathrm{M}-\mathrm{H}-\mathrm{CHO}]^{-}$and $108.0212[\mathrm{M}-\mathrm{H}-\mathrm{CH}]^{-}$. Peaks 36, 38, 46 and 57 had same molecular weight of $\mathrm{C}_{30} \mathrm{H}_{26} \mathrm{O}_{12}$, which illustrated that they were isomers. Base on the MS/MS spectra, peak 36 (407.0775 [M-H- ${ }_{8} \mathrm{H}_{8} \mathrm{O}_{3}-\mathrm{H}_{2} \mathrm{O}^{-}{ }^{-}, 289.0714$ [M-H- $\left.{ }_{15} \mathrm{H}_{13} \mathrm{O}_{6}\right]^{-}$), 38 (407.0768 [M$\left.\mathrm{H}_{-} \mathrm{C}_{8} \mathrm{H}_{8} \mathrm{O}_{3}-\mathrm{H}_{2} \mathrm{O}\right]^{-}, 289.0709$ [M-H-C $\left.\left._{15} \mathrm{H}_{13} \mathrm{O}_{6}\right]^{-}\right), 46\left(425.0893 \text { [M-H-C }_{8} \mathrm{H}_{8} \mathrm{O}_{3}\right]^{-}, 407.0782$ $\left.\left[\mathrm{M}-\mathrm{H}-\mathrm{C}_{8} \mathrm{H}_{8} \mathrm{O}_{3}-\mathrm{H}_{2} \mathrm{O}\right]^{-}, 289.0721\left[\mathrm{M}-\mathrm{H}-\mathrm{C}_{15} \mathrm{H}_{13} \mathrm{O}_{6}\right]^{-}\right)$and 57 (425.0886 [M-H-C $\left.{ }_{8} \mathrm{H}_{8} \mathrm{O}_{3}\right]^{-}$, 407.0789 [M-H-C $\left.{ }_{8} \mathrm{H}_{8} \mathrm{O}_{3}-\mathrm{H}_{2} \mathrm{O}\right]^{-}, 289.0734\left[\mathrm{M}-\mathrm{H}-\mathrm{C}_{15} \mathrm{H}_{13} \mathrm{O}_{6}\right]^{-}$) were detected, which could be identified as procyanidin B1 [41], procyanidin B3 [41], procyanidin B2 [41] and procyanidin B7 [41] by comparing the retention time of the reference standards and literatures. Peak 41 had a molecular weight of $289.0713\left(\mathrm{C}_{15} \mathrm{H}_{14} \mathrm{O}_{6}\right)$ with fragments of $245.0843\left[\mathrm{M}-\mathrm{H}-\mathrm{CO}_{2}\right]^{-}, 203.0713\left[\mathrm{M}-\mathrm{H}-\mathrm{H}_{2} \mathrm{O}-\mathrm{C}_{3} \mathrm{O}_{2}\right]^{-}$and $151.0401\left[\mathrm{M}-\mathrm{H}-\mathrm{C}_{8} \mathrm{H}_{10} \mathrm{O}_{2}\right]^{-}$, therefore it was identified as catechin [23]. Based on the same fragment, peaks 44 (289.0735 $\left.\left[\mathrm{M}-\mathrm{H}-\mathrm{C}_{7} \mathrm{H}_{2} \mathrm{O}_{4}\right]^{-}, 151.0386\left[\mathrm{M}-\mathrm{H}-\mathrm{C}_{7} \mathrm{H}_{4} \mathrm{O}_{4}-\mathrm{C}_{8} \mathrm{H}_{10} \mathrm{O}_{2}\right]^{-}\right), 51\left(203.0710\left[\mathrm{M}-\mathrm{H}-\mathrm{H}_{2} \mathrm{O}-\mathrm{C}_{3} \mathrm{O}_{2}\right]^{-}\right.$, 187.0396 [M-H-C $\left.{ }_{4} \mathrm{H}_{6} \mathrm{O}_{3}\right]^{-}$and $\left.151.0401\left[\mathrm{M}-\mathrm{H}-\mathrm{C}_{8} \mathrm{H}_{10} \mathrm{O}_{2}\right]^{-}\right), 58\left(316.0225\left[\mathrm{M}-\mathrm{H}_{-} \mathrm{C}_{6} \mathrm{H}_{11} \mathrm{O}_{5}\right]^{-}\right.$, 271.0249 [M-H- $\left._{6} \mathrm{H}_{11} \mathrm{O}_{5}-\mathrm{CO}_{-} \mathrm{H}_{2} \mathrm{O}\right]^{-}$and 151.0034 [M-H-C $\left.\left._{14} \mathrm{H}_{16} \mathrm{O}_{9}\right]^{-}\right), 64(285.0413$ [M$\left.\mathrm{H}-\mathrm{C}_{6} \mathrm{H}_{11} \mathrm{O}_{5}\right]^{-}$and 151.0041 [M-H-C $\left.\left.{ }_{14} \mathrm{H}_{8} \mathrm{O}_{7}\right]^{-}\right)$, $66\left(301.0352\left[\mathrm{M}-\mathrm{H}-\mathrm{C}_{6} \mathrm{H}_{10} \mathrm{O}_{5}\right]^{-}, 255.0296\right.$ $\left[\mathrm{M}-\mathrm{H}-\mathrm{C}_{6} \mathrm{H}_{10} \mathrm{O}_{5}-\mathrm{C}_{2} \mathrm{H}_{2} \mathrm{O}\right]^{-}$and $\left.151.0034\left[\mathrm{M}-\mathrm{H}-\mathrm{C}_{14} \mathrm{H}_{11} \mathrm{O}_{9}\right]^{-}\right), 67\left(330.0381\left[\mathrm{M}-\mathrm{H}_{-} \mathrm{C}_{6} \mathrm{H}_{11} \mathrm{O}_{5}\right]^{-}\right.$, 315.0155 [M-H-C $\left._{6} \mathrm{H}_{11} \mathrm{O}_{5}-\mathrm{CH}_{3}\right]^{-}, 271.0249\left[\mathrm{M}-\mathrm{H}-\mathrm{C}_{6} \mathrm{H}_{11} \mathrm{O}_{5}-\mathrm{CH}_{3}-\mathrm{CO}-\mathrm{H}_{2} \mathrm{O}\right]^{-}$and 151.0034 $\left.\left.\left[\mathrm{M}-\mathrm{H}-\mathrm{C}_{15} \mathrm{H}_{18} \mathrm{O}_{9}\right]^{-}\right), 68\left(151.0020 \text { [M-H-C }_{14} \mathrm{H}_{16} \mathrm{O}_{7}\right]^{-}\right), 69\left(285.0413 \text { [M-H-C }_{6} \mathrm{H}_{11} \mathrm{O}_{5}\right]^{-}$and 151.0041 [M-H-C $\left.\left.{ }_{14} \mathrm{H}_{8} \mathrm{O}_{7}\right]^{-}\right)$, 70 (301.0379 [M-H-C $\left.{ }_{6} \mathrm{H}_{10} \mathrm{O}_{5}\right]^{-}$and $151.0039\left[\mathrm{M}-\mathrm{H}-\mathrm{C}_{6} \mathrm{H}_{10} \mathrm{O}_{5^{-}}\right.$ $\left.\left.\mathrm{C}_{7} \mathrm{H}_{6} \mathrm{O}_{2}-\mathrm{CO}\right]^{-}\right), 72\left(301.0372 \text { [M-H-C }_{6} \mathrm{H}_{10} \mathrm{O}_{5}\right]^{-}, 255.0293\left[\mathrm{M}-\mathrm{H}-\mathrm{C}_{6} \mathrm{H}_{10} \mathrm{O}_{5}-\mathrm{C}_{2} \mathrm{H}_{2} \mathrm{O}\right]^{-}$and 151.0016 [M-H-C $\left.\left.{ }_{14} \mathrm{H}_{11} \mathrm{O}_{9}\right]^{-}\right)$, 73 (245.0504 [M-H-H $\left.\mathrm{H}_{2} \mathrm{O}-2 \mathrm{CO}\right]^{-}$and $\left.151.0035\left[\mathrm{M}-\mathrm{H}-\mathrm{C}_{8} \mathrm{H}_{6} \mathrm{O}_{4}\right]^{-}\right)$, $75\left(315.0530\left[\mathrm{M}-\mathrm{H}-\mathrm{C}_{6} \mathrm{H}_{10} \mathrm{O}_{5}\right]^{-}, 271.0255\left[\mathrm{M}-\mathrm{H}-\mathrm{C}_{8} \mathrm{H}_{14} \mathrm{O}_{6}\right]^{-}\right.$and $151.0034\left[\mathrm{M}-\mathrm{H}-\mathrm{C}_{15} \mathrm{H}_{18} \mathrm{O}_{8}\right]^{-}$),

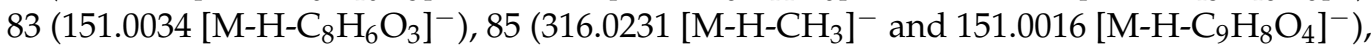

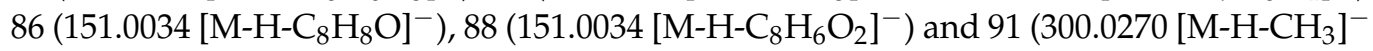
and $\left.151.0016\left[\mathrm{M}-\mathrm{H}-\mathrm{C}_{9} \mathrm{H}_{8} \mathrm{O}_{3}\right]^{-}\right)$were identified as the type of ion of $151.0037\left(\mathrm{C}_{7} \mathrm{H}_{4} \mathrm{O}_{4}\right)$, which were the characteristic fragment of flavonoid components. Indeed, they were epicatechin gallate [23], epicatechin [23], myricetin-3-O-galactoside [23], quercitrin [28], quercetin3-O-glucuronide [28], laricitrin-3-O-glucoside [37], luteolin-3'-glucoside [23], astilbin [38], isoquercitrin [22], astragalin [38], myricetin [21], isorhamnetin-3-O-glucoside [28], quercetin [22], laricitrin [37], naringenin [23], kaempferol [22] and isorhamnetin [28]. Peak 45 had the mass spectrum ion at $197.0454\left(\mathrm{C}_{9} \mathrm{H}_{10} \mathrm{O}_{5}\right)$ with MS/MS ions at 182.0256 [M-H- 
$\left.\mathrm{CH}_{3}\right]^{-}, 167.0014\left[\mathrm{M}-\mathrm{H}-\mathrm{CH}_{3}-\mathrm{CO}_{2}\right]^{-}, 138.0317\left[\mathrm{M}-\mathrm{H}-\mathrm{CH}_{3}-\mathrm{CO}_{2}-\mathrm{CH}_{3}\right]^{-}$and $123.0090[\mathrm{M}-\mathrm{H}-$ $\left.\mathrm{CH}_{3}-2 \mathrm{CO}_{2}-\mathrm{CH}_{3}\right]^{-}$, which can be identified as syringic acid [27]. Peak 47 produced [M-H] at $\mathrm{m} / \mathrm{z} 325.0926$ with the molecular formula $\mathrm{C}_{15} \mathrm{H}_{18} \mathrm{O}_{8}$. A loss of $\mathrm{C}_{6} \mathrm{H}_{10} \mathrm{O}_{5}$ in MS/MS was confirmed to be a hexose neutral loss. The other ions were 145.0294 [M- $\left.-\mathrm{H}_{6} \mathrm{H}_{10} \mathrm{O}_{5}-\mathrm{H}_{2} \mathrm{O}\right]^{-}$ and $117.0343\left[\mathrm{M}-\mathrm{H}-\mathrm{C}_{6} \mathrm{H}_{10} \mathrm{O}_{5}-\mathrm{CO}\right]^{-}$. Peak 47 was tentatively identified as p-coumaric acid glucoside [28]. Peak 48 had the major ions at $\mathrm{m} / \mathrm{z} 189.0769\left(\mathrm{C}_{8} \mathrm{H}_{14} \mathrm{O}_{5}\right)$ and the MS/MS fragment was $71.0503\left[\mathrm{M}-\mathrm{H}-\mathrm{C}_{2} \mathrm{H}_{5}-\mathrm{CO}_{2}-\mathrm{C}_{2} \mathrm{H}_{5} \mathrm{O}\right]^{-}$. Therefore, it was identified as diethyl malate [24]. Peak 53 was identified as ethyl gallate by its characteristic fragmentation pattern, which MS/MS spectra ions $\mathrm{m} / \mathrm{z} 169.0142\left[\mathrm{C}_{9} \mathrm{H}_{10} \mathrm{O}_{5}-\mathrm{C}_{2} \mathrm{H}_{4}\right]^{-}$and $125.0239\left[\mathrm{C}_{9} \mathrm{H}_{10} \mathrm{O}_{5^{-}}\right.$ $\left.\mathrm{C}_{2} \mathrm{H}_{4}-\mathrm{CO}_{2}\right]^{-}$were observed [28]. Peak 54 had the MS ion of 493.1341 and the MS/MS spectra ions of $\mathrm{m} / \mathrm{z} 331.0822\left[\mathrm{M}-\mathrm{H}-\mathrm{C}_{6} \mathrm{H}_{10} \mathrm{O}_{5}\right]^{-}$and $316.0553\left[\mathrm{M}-\mathrm{H}_{-} \mathrm{C}_{6} \mathrm{H}_{10} \mathrm{O}_{5}-\mathrm{CH}_{3}\right]^{-}$. Hence, peak 54 was tentatively identified as malvidin-3-O-glucoside [41]. By the similar regular pattern, peaks $62\left(303.0510\left[\mathrm{M}-\mathrm{H}-\mathrm{C}_{6} \mathrm{H}_{10} \mathrm{O}_{5}\right]^{-}\right.$and $316.0553\left[\mathrm{M}-\mathrm{H}-\mathrm{C}_{6} \mathrm{H}_{10} \mathrm{O}_{5}-2 \mathrm{H}_{2} \mathrm{O}-\right.$ $\left.\mathrm{CO}]^{-}\right), 65\left(303.0510\left[\mathrm{M}-\mathrm{H}-2 \mathrm{C}_{6} \mathrm{H}_{10} \mathrm{O}_{5}\right]^{-}\right), 74\left(317.0648\left[\mathrm{M}-\mathrm{H}_{-} \mathrm{C}_{6} \mathrm{H}_{10} \mathrm{O}_{5}\right]^{-}\right.$and $302.0429[\mathrm{M}-\mathrm{H}-$ $\left.\left.\mathrm{C}_{6} \mathrm{H}_{10} \mathrm{O}_{5}-\mathrm{CH}_{3}\right]^{-}\right), 78\left(303.0767\left[\mathrm{M}-\mathrm{H}-\mathrm{C}_{6} \mathrm{H}_{10} \mathrm{O}_{5}-\mathrm{CO}_{2}\right]^{-}\right)$and $80\left(477.1196\left[\mathrm{M}-\mathrm{H}-\mathrm{C}_{9} \mathrm{H}_{10} \mathrm{O}_{3}\right]^{-}\right.$ and $331.0874\left[\mathrm{M}-\mathrm{H}-\mathrm{C}_{9} \mathrm{H}_{10} \mathrm{O}_{3}-\mathrm{C}_{6} \mathrm{H}_{10} \mathrm{O}_{5}\right]^{-}$) were displayed in MS/MS spectra, which could be identified as delphinidin-3-O-glucoside [42,43], delphinidin-3,5-O-diglucoside [44], petunidin-3-O-glucoside [41], delphindin-3-O-(6-O-acetylglucoside) [44] and malvidin-3$\mathrm{O}-\left(6-\mathrm{O}\right.$-coumaroylglucoside) [44]. Peak 59 gave the $[\mathrm{M}-\mathrm{H}]^{-}$ion at the $\mathrm{m} / \mathrm{z} 389.1242$ with MS/MS spectra ions m/z 227.0994 [M- $\left.-\mathrm{H}_{-} \mathrm{C}_{6} \mathrm{H}_{10} \mathrm{O}_{5}\right]^{-}$and $143.0505\left[\mathrm{M}-\mathrm{H}-\mathrm{C}_{6} \mathrm{H}_{10} \mathrm{O}_{5}-\mathrm{C}_{2} \mathrm{H}_{2} \mathrm{O}-\right.$ $\mathrm{C}_{2} \mathrm{H}_{2} \mathrm{O}^{-}$. Peak 59 was tentatively identified as trans-piceid [23]. By the similar regular pattern, peaks $72\left(227.0994\left[\mathrm{M}-\mathrm{H}-\mathrm{C}_{6} \mathrm{H}_{10} \mathrm{O}_{5}\right]^{-}\right.$and $\left.185.0606\left[\mathrm{M}-\mathrm{H}-\mathrm{C}_{6} \mathrm{H}_{10} \mathrm{O}_{5}-\mathrm{C}_{2} \mathrm{H}_{2} \mathrm{O}\right]^{-}\right), 76$ (185.0587 [M-H- $\left.\mathrm{C}_{6} \mathrm{H}_{10} \mathrm{O}_{5}-\mathrm{C}_{2} \mathrm{H}_{2} \mathrm{O}\right]^{-}$and $\left.143.0501\left[\mathrm{M}-\mathrm{H}-\mathrm{C}_{6} \mathrm{H}_{10} \mathrm{O}_{5}-\mathrm{C}_{2} \mathrm{H}_{2} \mathrm{O}-\mathrm{C}_{2} \mathrm{H}_{2} \mathrm{O}\right]^{-}\right)$and $\left.84\left(143.0504 \text { [M- }-\mathrm{H}_{6} \mathrm{H}_{10} \mathrm{O}_{5}-\mathrm{C}_{2} \mathrm{H}_{2} \mathrm{O}-\mathrm{C}_{2} \mathrm{H}_{2} \mathrm{O}\right]^{-}\right)$were displayed in MS/MS spectra, which can be identified as cis-piceid [23], trans-resveratrol [23] and resveratrol [23] with their different retention times. Peak 63 showed the mass spectrum ion at $463.0884\left(\mathrm{C}_{21} \mathrm{H}_{20} \mathrm{O}_{12}\right)$. The product fragment ions were $301.0379\left[\mathrm{M}-\mathrm{H}-\mathrm{C}_{6} \mathrm{H}_{10} \mathrm{O}_{5}\right]^{-}, 300.0277\left[\mathrm{M}-\mathrm{H}-\mathrm{C}_{6} \mathrm{H}_{11} \mathrm{O}_{5}\right]^{-}$, 271.0245 [M-H- $\left.\mathrm{C}_{6} \mathrm{H}_{10} \mathrm{O}_{5}-\mathrm{CHO}\right]^{-}, 243.0291\left[\mathrm{M}-\mathrm{H}_{-} \mathrm{C}_{6} \mathrm{H}_{10} \mathrm{O}_{5}-\mathrm{CHO}_{2}\right]^{-}$and 151.0031 [M-H$\left.\mathrm{C}_{6} \mathrm{H}_{10} \mathrm{O}_{5}-\mathrm{C}_{7} \mathrm{H}_{6} \mathrm{O}_{2}-\mathrm{CO}\right]^{-}$. Therefore, it was identified as hyperoside [23]. Peak 77 gave a mass spectrum ion at $435.1300\left(\mathrm{C}_{21} \mathrm{H}_{24} \mathrm{O}_{10}\right)$ with MS/MS fragments of 273.0769 [M-H$\left.\mathrm{C}_{6} \mathrm{H}_{12} \mathrm{O}_{6}\right]^{-}, 179.0349$ [M-H- $\left.\mathrm{C}_{15} \mathrm{H}_{14} \mathrm{O}_{5}\right]^{-}$and 167.0352 [M-H- $\left.\mathrm{C}_{6} \mathrm{H}_{12} \mathrm{O}_{6}-\mathrm{C}_{7} \mathrm{H}_{6} \mathrm{O}\right]^{-}$, which was detected to be phloridzin [45]. Peak 81 had a molecular weight of $303.0497\left(\mathrm{C}_{15} \mathrm{H}_{10} \mathrm{O}_{7}\right)$ with fragments of $285.0398\left[\mathrm{M}+\mathrm{H}-\mathrm{H}_{2} \mathrm{O}\right]^{+}, 257.0456\left[\mathrm{M}+\mathrm{H}-\mathrm{H}_{2} \mathrm{O}-\mathrm{CO}\right]^{+}, 229.0492\left[\mathrm{M}+\mathrm{H}-\mathrm{H}_{2} \mathrm{O}-\right.$ $2 \mathrm{CO}]^{+}$and $153.0188\left[\mathrm{M}+\mathrm{H}-\mathrm{C}_{8} \mathrm{H}_{5} \mathrm{O}_{3}\right]^{+}$, therefore it was identified as morin [46]. Peak 82 had the mass spectrum ion at 303.0497 and MS/MS experiment gave fragmentations information at $229.0492\left[\mathrm{M}-\mathrm{H}-\mathrm{CO}-2 \mathrm{H}_{2} \mathrm{O}\right]^{-}$and $153.0188\left[\mathrm{M}-\mathrm{H}-\mathrm{C}_{8} \mathrm{H}_{6} \mathrm{O}_{3}\right]^{-}$, which can be identified as delphinidin [28]. By the similar regular pattern, peaks $87\left(213.0559\left[\mathrm{M}-\mathrm{H}-2 \mathrm{H}_{2} \mathrm{O}-\mathrm{CO}\right]\right.$ and $\left.137.0233\left[\mathrm{M}-\mathrm{H}-\mathrm{C}_{8} \mathrm{H}_{6} \mathrm{O}_{3}\right]\right)$ and $90\left(229.0498\left[\mathrm{M}-\mathrm{H}-2 \mathrm{H}_{2} \mathrm{O}-\mathrm{CO}-\mathrm{CH}_{3}\right]\right.$ and $153.0188[\mathrm{M}-\mathrm{H}-$ $\mathrm{CH}_{3}-\mathrm{C}_{8} \mathrm{H}_{6} \mathrm{O}_{3}$ ]) were showed in MS/MS spectra, which can be identified as cyanidin [28] and petunidin [28]. Peak 89 was identified as syringetin with MS/MS spectra ions $\mathrm{m} / \mathrm{z}$ $330.0385\left[\mathrm{M}-\mathrm{H}-\mathrm{CH}_{3}\right]^{-}$and $315.0153\left[\mathrm{M}-\mathrm{H}_{-} \mathrm{C}_{2} \mathrm{H}_{6}\right]^{-}$[47]. Peak 92, the molecular weight was $193.1588\left(\mathrm{C}_{13} \mathrm{H}_{20} \mathrm{O}\right)$ and the fragment ion was observed at $\mathrm{m} / \mathrm{z} 56.9419\left[\mathrm{M}+\mathrm{H}-\mathrm{C}_{10} \mathrm{H}_{15}\right]^{+}$, which was identified as ionone [48]. Peak 93 had a molecular weight of $173.1536\left(\mathrm{C}_{10} \mathrm{H}_{20} \mathrm{O}_{2}\right)$ with fragment of $76.0221[\mathrm{M}+\mathrm{H}-\mathrm{HCOOH}]^{+}$, therefore it was identified as ethyl caprylate $\left(\mathrm{C}_{10} \mathrm{H}_{20} \mathrm{O}_{2}\right)$. The molecular weight of peak 94 was 195.1748. MS/MS ions of $\mathrm{m} / \mathrm{z} 177.1634$ $\left[\mathrm{M}+\mathrm{H}-\mathrm{H}_{2} \mathrm{O}\right]^{+}$and $163.0394\left[\mathrm{M}+\mathrm{H}-\mathrm{H}_{2} \mathrm{O}-\mathrm{CH}_{2}\right]^{+}$were observed, which revealed that it was theaspirane. Moreover, the MS spectrums of all compounds were shown in Supplementary Materials (Figures S1-S94). 
Table 2. UFLC/Q-TOF-MS data and identification 94 compounds from red wine.

\begin{tabular}{|c|c|c|c|c|c|c|c|}
\hline Peak No. & $\begin{array}{c}\mathrm{RT} \\
(\mathrm{min})\end{array}$ & Ion & $\begin{array}{l}\text { Found at } \\
\text { Mass }\end{array}$ & $\begin{array}{c}\text { MS/MS } \\
\text { Fragmentation Ions }\end{array}$ & Formula & Identification & ppm \\
\hline 1 & 0.70 & {$[\mathrm{M}+\mathrm{H}]^{+}$} & 116.0702 & 70.0647 & $\mathrm{C}_{5} \mathrm{H}_{9} \mathrm{NO}_{2}$ & D-proline & 0.8 \\
\hline 2 & 0.76 & {$[\mathrm{M}+\mathrm{H}]^{+}$} & 175.1189 & 70.0652 & $\mathrm{C}_{6} \mathrm{H}_{14} \mathrm{~N}_{4} \mathrm{O}_{2}$ & L-arginine & -0.3 \\
\hline 3 & 0.79 & {$[\mathrm{M}-\mathrm{H}]^{-}$} & 179.0562 & $\begin{array}{c}99.0087,71.0137 \\
59.0139\end{array}$ & $\mathrm{C}_{6} \mathrm{H}_{12} \mathrm{O}_{6}$ & D-glucose & 0.3 \\
\hline 4 & 0.88 & {$[\mathrm{M}-\mathrm{H}]^{-}$} & 181.0720 & $\begin{array}{c}83.0143,71.0140 \\
59.0140\end{array}$ & $\mathrm{C}_{6} \mathrm{H}_{14} \mathrm{O}_{6}$ & D-mannitol & 1.5 \\
\hline 5 & 0.96 & {$[\mathrm{M}-\mathrm{H}]^{-}$} & 191.0199 & $85.0296,59.0134$ & $\mathrm{C}_{6} \mathrm{H}_{8} \mathrm{O}_{7}$ & citric acid & 1.0 \\
\hline 6 & 0.99 & {$[\mathrm{M}-\mathrm{H}]^{-}$} & 149.0091 & $\begin{array}{c}105.0217,87.0085 \\
72.9928\end{array}$ & $\mathrm{C}_{4} \mathrm{H}_{6} \mathrm{O}_{6}$ & tartaric acid & -0.7 \\
\hline 7 & 1.04 & {$[\mathrm{M}+\mathrm{H}]^{+}$} & 138.0548 & $\begin{array}{c}120.0433,92.0495 \\
78.0339\end{array}$ & $\mathrm{C}_{7} \mathrm{H}_{7} \mathrm{NO}_{2}$ & 4-aminobenzoic acid & -1.5 \\
\hline 8 & 1.12 & {$[\mathrm{M}-\mathrm{H}]^{-}$} & 341.1088 & $\begin{array}{c}161.0459,71.0137 \\
59.0138\end{array}$ & $\mathrm{C}_{12} \mathrm{H}_{22} \mathrm{O}_{11}$ & trehalose & -0.3 \\
\hline 9 & 1.15 & {$[\mathrm{M}-\mathrm{H}]^{-}$} & 149.0455 & $75.0221,59.0133$ & $\mathrm{C}_{5} \mathrm{H}_{10} \mathrm{O}_{5}$ & D-ribose & -0.2 \\
\hline 10 & 1.31 & {$[\mathrm{M}-\mathrm{H}]^{-}$} & 133.0142 & $115.0018,71.0136$ & $\mathrm{C}_{4} \mathrm{H}_{6} \mathrm{O}_{5}$ & L-malic acid & -0.5 \\
\hline 11 & 1.33 & {$[\mathrm{M}+\mathrm{H}]^{+}$} & 118.0862 & 72.0809 & $\mathrm{C}_{5} \mathrm{H}_{11} \mathrm{NO}_{2}$ & L-valine & -0.4 \\
\hline 12 & 1.48 & {$[\mathrm{M}-\mathrm{H}]^{-}$} & 173.0454 & $137.0225,93.0343$ & $\mathrm{C}_{7} \mathrm{H}_{10} \mathrm{O}_{5}$ & shikimic acid & -1.0 \\
\hline 13 & 1.71 & {$[\mathrm{M}+\mathrm{H}]^{+}$} & 150.0586 & $74.0243,61.0108$ & $\mathrm{C}_{5} \mathrm{H}_{11} \mathrm{NO}_{2} \mathrm{~S}$ & L-methionine & 1.8 \\
\hline 14 & 2.03 & {$[\mathrm{M}+\mathrm{H}]^{+}$} & 124.0393 & $80.0506,55.9346$ & $\mathrm{C}_{6} \mathrm{H}_{5} \mathrm{NO}_{2}$ & niacin & 0.2 \\
\hline 15 & 3.27 & {$[\mathrm{M}-\mathrm{H}]^{-}$} & 117.0192 & $99.9254,73.0291$ & $\mathrm{C}_{4} \mathrm{H}_{6} \mathrm{O}_{4}$ & succinic acid & -1.2 \\
\hline 16 & 3.51 & {$[\mathrm{M}+\mathrm{H}]^{+}$} & 165.0547 & $\begin{array}{c}119.0479,95.0503 \\
77.0388,59.9302\end{array}$ & $\mathrm{C}_{9} \mathrm{H}_{8} \mathrm{O}_{3}$ & $\begin{array}{l}\text { trans-4- } \\
\text { hydroxycinnamic } \\
\text { acid }\end{array}$ & 0.7 \\
\hline 17 & 3.85 & {$[\mathrm{M}+\mathrm{H}]^{+}$} & 137.0913 & $\begin{array}{c}\text { 121.0616, 103.0616, } \\
95.0561,91.0556 \\
77.0391\end{array}$ & $\mathrm{C}_{8} \mathrm{H}_{11} \mathrm{NO}$ & tyramine & -0.5 \\
\hline 18 & 5.97 & {$[\mathrm{M}-\mathrm{H}]^{-}$} & 169.0141 & $\begin{array}{c}125.0244,107.0156 \\
79.0186\end{array}$ & $\mathrm{C}_{7} \mathrm{H}_{6} \mathrm{O}_{5}$ & gallic acid & -1.0 \\
\hline 19 & 8.77 & {$[\mathrm{M}+\mathrm{H}]^{+}$} & 141.0182 & $\begin{array}{c}123.0080,95.0127 \\
67.0187,55.9453\end{array}$ & $\mathrm{C}_{6} \mathrm{H}_{4} \mathrm{O}_{4}$ & coumalic acid & -0.1 \\
\hline 20 & 11.49 & {$[\mathrm{M}-\mathrm{H}]^{-}$} & 153.0558 & $\begin{array}{c}123.0085,108.0221 \\
95.0139,78.9590\end{array}$ & $\mathrm{C}_{8} \mathrm{H}_{10} \mathrm{O}_{3}$ & hydroxytyrosol & 0.2 \\
\hline 21 & 11.57 & {$[\mathrm{M}-\mathrm{H}]^{-}$} & 153.0194 & $\begin{array}{c}109.0298,91.0298 \\
65.0029\end{array}$ & $\mathrm{C}_{7} \mathrm{H}_{6} \mathrm{O}_{4}$ & protocatechuic acid & 0.3 \\
\hline 22 & 13.85 & {$[\mathrm{M}-\mathrm{H}]^{-}$} & 305.0664 & $\begin{array}{l}179.0360,165.0194 \\
137.0243,125.0246\end{array}$ & $\mathrm{C}_{15} \mathrm{H}_{14} \mathrm{O}_{7}$ & $(+)$-gallocatechin & -1.0 \\
\hline 23 & 14.27 & {$[\mathrm{M}-\mathrm{H}]^{-}$} & 179.0349 & $\begin{array}{c}135.0560,89.0394 \\
79.0550\end{array}$ & $\mathrm{C}_{9} \mathrm{H}_{8} \mathrm{O}_{4}$ & acetylsalicylic acid & -0.7 \\
\hline 24 & 14.27 & {$[\mathrm{M}-\mathrm{H}]^{-}$} & 179.0348 & $\begin{array}{c}161.0263,135.0454 \\
117.0351\end{array}$ & $\mathrm{C}_{13} \mathrm{H}_{12} \mathrm{O}_{9}$ & caftaric acid & -1.0 \\
\hline 25 & 15.05 & {$[\mathrm{M}-\mathrm{H}]^{-}$} & 153.0194 & $\begin{array}{c}123.0061,109.0298 \\
91.0298\end{array}$ & $\mathrm{C}_{7} \mathrm{H}_{6} \mathrm{O}_{4}$ & gentisic acid & 0.4 \\
\hline 26 & 15.21 & {$[\mathrm{M}-\mathrm{H}]^{-}$} & 137.0255 & 108.0213 & $\mathrm{C}_{7} \mathrm{H}_{6} \mathrm{O}_{3}$ & protocatechualdehyde & -2.7 \\
\hline 27 & 15.31 & {$[\mathrm{M}-\mathrm{H}]^{-}$} & 181.0506 & 135.0453 & $\mathrm{C}_{9} \mathrm{H}_{10} \mathrm{O}_{4}$ & homovanillic acid & 0.0 \\
\hline 28 & 15.43 & {$[\mathrm{M}-\mathrm{H}]^{-}$} & 183.0299 & $124.0234,95.0136$ & $\mathrm{C}_{8} \mathrm{H}_{8} \mathrm{O}_{5}$ & methyl gallate & 0.0 \\
\hline 29 & 15.90 & {$[\mathrm{M}-\mathrm{H}]^{-}$} & 137.0244 & 93.0340 & $\mathrm{C}_{7} \mathrm{H}_{6} \mathrm{O}_{3}$ & $\begin{array}{l}\text { 4-hydroxybenzoic } \\
\text { acid }\end{array}$ & 0.4 \\
\hline 30 & 16.32 & {$[\mathrm{M}-\mathrm{H}]^{-}$} & 353.0877 & $\begin{array}{c}191.0616,179.0341 \\
135.0439\end{array}$ & $\mathrm{C}_{16} \mathrm{H}_{18} \mathrm{O}_{9}$ & chlorogenic acid & -0.2 \\
\hline 31 & 17.36 & {$[\mathrm{M}-\mathrm{H}]^{-}$} & 295.0458 & $\begin{array}{c}163.0400,119.0503 \\
87.0094\end{array}$ & $\mathrm{C}_{13} \mathrm{H}_{12} \mathrm{O}_{8}$ & p-coutaric acid & 0.1 \\
\hline 32 & 18.08 & {$[\mathrm{M}-\mathrm{H}]^{-}$} & 163.0401 & $119.0498,93.0347$ & $\mathrm{C}_{9} \mathrm{H}_{8} \mathrm{O}_{3}$ & m-coumaric acid & 0.2 \\
\hline 33 & 18.08 & {$[\mathrm{M}-\mathrm{H}]^{-}$} & 295.0458 & $163.0400,119.0498$ & $\mathrm{C}_{13} \mathrm{H}_{12} \mathrm{O}_{8}$ & coutaric acid & -0.4 \\
\hline 34 & 18.15 & {$[\mathrm{M}-\mathrm{H}]^{-}$} & 145.0507 & $73.0273,55.0191$ & $\mathrm{C}_{6} \mathrm{H}_{10} \mathrm{O}_{4}$ & dimethyl succinate & 0.6 \\
\hline
\end{tabular}


Table 2. Cont.

\begin{tabular}{|c|c|c|c|c|c|c|c|}
\hline Peak No. & $\begin{array}{c}\text { RT } \\
(\min )\end{array}$ & Ion & $\begin{array}{c}\text { Found at } \\
\text { Mass }\end{array}$ & $\begin{array}{c}\text { MS/MS } \\
\text { Fragmentation Ions }\end{array}$ & Formula & Identification & ppm \\
\hline 35 & 19.24 & {$[\mathrm{M}-\mathrm{H}]^{-}$} & 121.0296 & $\begin{array}{c}108.0212,92.0265 \\
425.0864,407.0775\end{array}$ & $\mathrm{C}_{7} \mathrm{H}_{6} \mathrm{O}_{2}$ & benzoic acid & 0.8 \\
\hline 36 & 19.66 & {$[\mathrm{M}-\mathrm{H}]^{-}$} & 577.1346 & $\begin{array}{c}289.0714,161.0238 \\
125.0243\end{array}$ & $\mathrm{C}_{30} \mathrm{H}_{26} \mathrm{O}_{12}$ & procyanidin B1 & -0.9 \\
\hline 37 & 19.89 & {$[\mathrm{M}-\mathrm{H}]^{-}$} & 305.0664 & $\begin{array}{l}245.0462,219.0652 \\
139.0401,125.0244\end{array}$ & $\mathrm{C}_{15} \mathrm{H}_{14} \mathrm{O}_{7}$ & (-)-epigallocatechin & 0.2 \\
\hline 38 & 19.98 & {$[\mathrm{M}-\mathrm{H}]^{-}$} & 577.1346 & $407.0768,289.0709$ & $\mathrm{C}_{30} \mathrm{H}_{26} \mathrm{O}_{12}$ & procyanidin B3 & 0.0 \\
\hline 39 & 20.01 & {$[\mathrm{M}-\mathrm{H}]^{-}$} & 167.0350 & 108.0212 & $\mathrm{C}_{8} \mathrm{H}_{8} \mathrm{O}_{4}$ & vanillic acid & 0.2 \\
\hline 40 & 20.21 & {$[\mathrm{M}-\mathrm{H}]^{-}$} & 163.0400 & $119.0502,93.0343$ & $\mathrm{C}_{9} \mathrm{H}_{8} \mathrm{O}_{3}$ & $\begin{array}{l}\text { 2-Hydroxycinnamic } \\
\text { acid }\end{array}$ & -0.1 \\
\hline 41 & 20.47 & {$[\mathrm{M}-\mathrm{H}]^{-}$} & 289.0713 & $\begin{array}{l}245.0843,203.0713 \\
151.0401,109.0294\end{array}$ & $\mathrm{C}_{15} \mathrm{H}_{14} \mathrm{O}_{6}$ & catechin & -1.5 \\
\hline 42 & 20.58 & {$[\mathrm{M}-\mathrm{H}]^{-}$} & 325.0563 & $193.0490,117.0341$ & $\mathrm{C}_{14} \mathrm{H}_{14} \mathrm{O}_{9}$ & fertaric acid & -0.5 \\
\hline 43 & 20.98 & {$[\mathrm{M}-\mathrm{H}]^{-}$} & 179.0349 & $135.0449,89.0393$ & $\mathrm{C}_{9} \mathrm{H}_{8} \mathrm{O}_{4}$ & caffeic acid & -1.0 \\
\hline 44 & 21.90 & {$[\mathrm{M}-\mathrm{H}]^{-}$} & 441.0186 & $\begin{array}{l}289.0735,217.0167 \\
191.0337,151.0386\end{array}$ & $\mathrm{C}_{22} \mathrm{H}_{18} \mathrm{O}_{10}$ & epicatechin gallate & 2.2 \\
\hline 45 & 22.06 & {$[\mathrm{M}-\mathrm{H}]^{-}$} & 197.0454 & $\begin{array}{c}182.0256,167.0014 \\
138.0317,123.0090 \\
95.0142\end{array}$ & $\mathrm{C}_{9} \mathrm{H}_{10} \mathrm{O}_{5}$ & syringic acid & -0.3 \\
\hline 46 & 23.01 & {$[\mathrm{M}-\mathrm{H}]^{-}$} & 577.1346 & $\begin{array}{c}425.0893,407.0782 \\
289.0721\end{array}$ & $\mathrm{C}_{30} \mathrm{H}_{26} \mathrm{O}_{12}$ & procyanidin B2 & -0.7 \\
\hline 47 & 23.11 & {$[\mathrm{M}-\mathrm{H}]^{-}$} & 325.0926 & $\begin{array}{c}163.0394,145.0294 \\
117.0343\end{array}$ & $\mathrm{C}_{15} \mathrm{H}_{18} \mathrm{O}_{8}$ & $\begin{array}{l}\text { p-coumaric acid } \\
\text { glucoside }\end{array}$ & -0.5 \\
\hline 48 & 23.37 & {$[\mathrm{M}-\mathrm{H}]^{-}$} & 189.0769 & 71.0503 & $\mathrm{C}_{8} \mathrm{H}_{14} \mathrm{O}_{5}$ & diethyl malate & 0.3 \\
\hline 49 & 23.69 & {$[\mathrm{M}-\mathrm{H}]^{-}$} & 165.0556 & $119.0527,103.0565$ & $\mathrm{C}_{9} \mathrm{H}_{10} \mathrm{O}_{3}$ & desaminotyrosine & -0.5 \\
\hline 50 & 24.37 & {$[\mathrm{M}-\mathrm{H}]^{-}$} & 137.0244 & $93.0470,65.0473$ & $\mathrm{C}_{7} \mathrm{H}_{6} \mathrm{O}_{3}$ & $\begin{array}{l}\text { 2-hydroxybenzoic } \\
\text { acid }\end{array}$ & 2.2 \\
\hline 51 & 24.38 & {$[\mathrm{M}-\mathrm{H}]^{-}$} & 289.0713 & $\begin{array}{c}\text { 203.0710, 187.0396, } \\
151.0401,123.0449 \\
109.0290\end{array}$ & $\mathrm{C}_{15} \mathrm{H}_{14} \mathrm{O}_{6}$ & epicatechin & -0.7 \\
\hline 52 & 24.68 & {$[\mathrm{M}-\mathrm{H}]^{-}$} & 167.0350 & $123.0436,108.0212$ & $\mathrm{C}_{8} \mathrm{H}_{8} \mathrm{O}_{4}$ & $\begin{array}{l}\text { 3-hydroxy-4- } \\
\text { methoxybenzoic } \\
\text { acid }\end{array}$ & 2.5 \\
\hline 53 & 25.16 & {$[\mathrm{M}-\mathrm{H}]^{-}$} & 197.0454 & $\begin{array}{c}169.0142,125.0239 \\
79.0184\end{array}$ & $\mathrm{C}_{9} \mathrm{H}_{10} \mathrm{O}_{5}$ & ethyl gallate & -0.9 \\
\hline 54 & 25.32 & {$[\mathrm{M}]^{+}$} & 493.1341 & $331.0822,316.0553$ & $\mathrm{C}_{23} \mathrm{H}_{25} \mathrm{O}_{12}$ & $\begin{array}{l}\text { malvidin-3-O- } \\
\text { glucoside }\end{array}$ & 0.1 \\
\hline 55 & 25.74 & {$[\mathrm{M}-\mathrm{H}]^{-}$} & 163.0401 & $119.0503,93.0347$ & $\mathrm{C}_{9} \mathrm{H}_{8} \mathrm{O}_{3}$ & p-coumaric acid & -0.6 \\
\hline 56 & 30.75 & {$[\mathrm{M}-\mathrm{H}]^{-}$} & 167.0350 & $123.0450,65.0024$ & $\mathrm{C}_{8} \mathrm{H}_{8} \mathrm{O}_{4}$ & homogentisic acid & 0.8 \\
\hline 57 & 30.80 & {$[\mathrm{M}-\mathrm{H}]^{-}$} & 577.1346 & $\begin{array}{c}425.0886,407.0789 \\
289.0734\end{array}$ & $\mathrm{C}_{30} \mathrm{H}_{26} \mathrm{O}_{12}$ & procyanidin B7 & 0.6 \\
\hline 58 & 34.06 & {$[\mathrm{M}-\mathrm{H}]^{-}$} & 479.0826 & $\begin{array}{c}316.0225,271.0249 \\
151.0034\end{array}$ & $\mathrm{C}_{21} \mathrm{H}_{20} \mathrm{O}_{13}$ & $\begin{array}{l}\text { myricetin-3-O- } \\
\text { galactoside }\end{array}$ & -1.0 \\
\hline 59 & 35.93 & {$[\mathrm{M}-\mathrm{H}]^{-}$} & 389.1242 & $\begin{array}{c}227.0716,185.0606 \\
143.0505\end{array}$ & $\mathrm{C}_{20} \mathrm{H}_{22} \mathrm{O}_{8}$ & trans-piceid & -0.2 \\
\hline 60 & 38.70 & {$[\mathrm{M}-\mathrm{H}]^{-}$} & 300.9987 & $\begin{array}{c}257.011,229.0126 \\
145.0295\end{array}$ & $\mathrm{C}_{14} \mathrm{H}_{6} \mathrm{O}_{8}$ & ellagic acid & -0.9 \\
\hline 61 & 38.84 & {$[\mathrm{M}-\mathrm{H}]^{-}$} & 181.0506 & $109.0286,65.0032$ & $\mathrm{C}_{9} \mathrm{H}_{10} \mathrm{O}_{4}$ & dihydrocaffeic acid & 0.5 \\
\hline 62 & 39.28 & {$[\mathrm{M}]^{+}$} & 465.1036 & $303.051,229.0488$ & $\mathrm{C}_{21} \mathrm{H}_{21} \mathrm{O}_{12}$ & $\begin{array}{l}\text { delphinidin-3-O- } \\
\text { glucoside }\end{array}$ & 1.9 \\
\hline 63 & 39.31 & {$[\mathrm{M}-\mathrm{H}]^{-}$} & 463.0884 & $\begin{array}{l}301.0379,271.0245 \\
243.0291,151.0031\end{array}$ & $\mathrm{C}_{21} \mathrm{H}_{20} \mathrm{O}_{12}$ & hyperoside & 0.4 \\
\hline 64 & 39.71 & {$[\mathrm{M}-\mathrm{H}]^{-}$} & 449.1090 & $285.0413,151.0041$ & $\mathrm{C}_{21} \mathrm{H}_{22} \mathrm{O}_{11}$ & quercitrin & 0.2 \\
\hline
\end{tabular}


Table 2. Cont.

\begin{tabular}{|c|c|c|c|c|c|c|c|}
\hline Peak No. & $\begin{array}{c}\mathrm{RT} \\
(\mathrm{min})\end{array}$ & Ion & $\begin{array}{l}\text { Found at } \\
\text { Mass }\end{array}$ & $\begin{array}{c}\text { MS/MS } \\
\text { Fragmentation Ions }\end{array}$ & Formula & Identification & ppm \\
\hline 65 & 39.83 & {$[\mathrm{M}]^{+}$} & 627.1462 & 303.0351 & $\mathrm{C}_{27} \mathrm{H}_{31} \mathrm{O}_{17}$ & $\begin{array}{l}\text { delphinidin-3,5-O- } \\
\text { diglucoside }\end{array}$ & 1.1 \\
\hline 66 & 40.08 & {$[\mathrm{M}-\mathrm{H}]^{-}$} & 477.0670 & $\begin{array}{c}301.0352,255.0296 \\
151.0034\end{array}$ & $\mathrm{C}_{21} \mathrm{H}_{18} \mathrm{O}_{13}$ & $\begin{array}{l}\text { quercetin-3-O- } \\
\text { glucuronide }\end{array}$ & -0.9 \\
\hline 67 & 41.28 & {$[\mathrm{M}-\mathrm{H}]^{-}$} & 493.0985 & $\begin{array}{l}330.0381,315.0155 \\
271.0249,151.0034\end{array}$ & $\mathrm{C}_{22} \mathrm{H}_{22} \mathrm{O}_{13}$ & $\begin{array}{l}\text { laricitrin-3-O- } \\
\text { glucoside }\end{array}$ & -0.6 \\
\hline 68 & 42.86 & {$[\mathrm{M}-\mathrm{H}]^{-}$} & 447.0936 & $\begin{array}{l}301.0372,271.0256, \\
255.0295,151.0020\end{array}$ & $\mathrm{C}_{21} \mathrm{H}_{20} \mathrm{O}_{11}$ & luteolin $-3^{\prime}$-glucoside & 1.2 \\
\hline 69 & 42.98 & {$[\mathrm{M}-\mathrm{H}]^{-}$} & 449.1090 & $285.0413,151.0041$ & $\mathrm{C}_{21} \mathrm{H}_{22} \mathrm{O}_{11}$ & astilbin & 0.9 \\
\hline 70 & 43.45 & {$[\mathrm{M}-\mathrm{H}]^{-}$} & 463.0884 & $\begin{array}{c}301.0379,255.0305 \\
151.0039\end{array}$ & $\mathrm{C}_{21} \mathrm{H}_{20} \mathrm{O}_{12}$ & isoquercitrin & 0.8 \\
\hline 71 & 44.78 & {$[\mathrm{M}-\mathrm{H}]^{-}$} & 447.0936 & $\begin{array}{c}301.0372,255.0293 \\
151.0016\end{array}$ & $\mathrm{C}_{21} \mathrm{H}_{20} \mathrm{O}_{11}$ & astragalin & 0.8 \\
\hline 72 & 45.19 & {$[\mathrm{M}-\mathrm{H}]^{-}$} & 389.1242 & $227.0994,185.0606$ & $\mathrm{C}_{20} \mathrm{H}_{22} \mathrm{O}_{8}$ & cis-piceid & 0.0 \\
\hline 73 & 45.72 & {$[\mathrm{M}-\mathrm{H}]^{-}$} & 317.0300 & $\begin{array}{l}245.0504,137.0241, \\
109.0292,151.0035\end{array}$ & $\mathrm{C}_{15} \mathrm{H}_{10} \mathrm{O}_{8}$ & myricetin & -0.9 \\
\hline 74 & 45.77 & {$[\mathrm{M}]^{+}$} & 479.1193 & $\begin{array}{l}317.0648,302.0429 \\
285.0362,153.0184\end{array}$ & $\mathrm{C}_{22} \mathrm{H}_{23} \mathrm{O}_{12}$ & $\begin{array}{l}\text { petunidin-3-O- } \\
\text { glucoside }\end{array}$ & 1.9 \\
\hline 75 & 45.80 & {$[\mathrm{M}-\mathrm{H}]^{-}$} & 477.1041 & $\begin{array}{c}315.0530,300.0263 \\
271.0255,243.0306 \\
151.0036\end{array}$ & $\mathrm{C}_{22} \mathrm{H}_{22} \mathrm{O}_{12}$ & $\begin{array}{l}\text { isorhamnetin-3-O- } \\
\text { glucoside }\end{array}$ & 0.6 \\
\hline 76 & 47.17 & {$[\mathrm{M}-\mathrm{H}]^{-}$} & 227.0715 & $\begin{array}{c}185.0587,143.0501 \\
115.0556\end{array}$ & $\mathrm{C}_{14} \mathrm{H}_{12} \mathrm{O}_{3}$ & trans-resveratrol & 0.6 \\
\hline 77 & 47.42 & {$[\mathrm{M}-\mathrm{H}]^{-}$} & 435.1300 & $\begin{array}{c}273.0769,179.0349 \\
167.0352\end{array}$ & $\mathrm{C}_{21} \mathrm{H}_{24} \mathrm{O}_{10}$ & phloridzin & 0.7 \\
\hline 78 & 49.16 & {$[\mathrm{M}]^{+}$} & 507.1141 & $\begin{array}{c}\text { 303.0767, 187.0626, } \\
113.0234\end{array}$ & $\mathrm{C}_{23} \mathrm{H}_{23} \mathrm{O}_{13}$ & $\begin{array}{l}\text { delphindin-3-O-(6- } \\
\text { O-acetylglucoside) }\end{array}$ & 1.6 \\
\hline 79 & 51.13 & {$[\mathrm{M}-\mathrm{H}]^{-}$} & 207.0662 & $179.0433,135.0446$ & $\mathrm{C}_{11} \mathrm{H}_{12} \mathrm{O}_{4}$ & ethyl caffeate & -0.6 \\
\hline 80 & 51.94 & {$[\mathrm{M}]^{+}$} & 639.1719 & $\begin{array}{c}\text { 477.1176, } 419.0853 \\
331.0874\end{array}$ & $\mathrm{C}_{32} \mathrm{H}_{31} \mathrm{O}_{14}$ & $\begin{array}{l}\text { malvidin-3-O-(6-O- } \\
\text { coumaroylglucoside) }\end{array}$ & 1.7 \\
\hline 81 & 52.60 & {$[\mathrm{M}+\mathrm{H}]^{+}$} & 303.0497 & $\begin{array}{l}285.0398,257.0456, \\
229.0492,153.0188\end{array}$ & $\mathrm{C}_{15} \mathrm{H}_{10} \mathrm{O}_{7}$ & morin & -0.8 \\
\hline 82 & 52.60 & {$[\mathrm{M}]^{+}$} & 303.0497 & $229.0492,153.0188$ & $\mathrm{C}_{15} \mathrm{H}_{11} \mathrm{O}_{7}$ & delphinidin & -0.8 \\
\hline 83 & 52.63 & {$[\mathrm{M}-\mathrm{H}]^{-}$} & 301.0351 & $\begin{array}{l}245.0447,229.0513, \\
151.0034,121.0292\end{array}$ & $\mathrm{C}_{15} \mathrm{H}_{10} \mathrm{O}_{7}$ & quercetin & -1.0 \\
\hline 84 & 52.67 & {$[\mathrm{M}-\mathrm{H}]^{-}$} & 227.0715 & 143.0504 & $\mathrm{C}_{14} \mathrm{H}_{12} \mathrm{O}_{3}$ & resveratrol & -0.9 \\
\hline 85 & 53.23 & {$[\mathrm{M}-\mathrm{H}]^{-}$} & 331.0459 & $\begin{array}{l}316.0231,271.0251, \\
178.9991,151.0039\end{array}$ & $\mathrm{C}_{16} \mathrm{H}_{12} \mathrm{O}_{8}$ & laricitrin & 0.0 \\
\hline 86 & 55.82 & {$[\mathrm{M}-\mathrm{H}]^{-}$} & 271.0613 & $\begin{array}{l}229.0500,177.0190 \\
151.0048,119.0618\end{array}$ & $\mathrm{C}_{15} \mathrm{H}_{12} \mathrm{O}_{5}$ & naringenin & 0.4 \\
\hline 87 & 57.10 & {$[\mathrm{M}]^{+}$} & 287.0549 & $\begin{array}{c}213.0559,153.0180 \\
137.0233\end{array}$ & $\mathrm{C}_{15} \mathrm{H}_{11} \mathrm{O}_{6}$ & cyanidin & -0.3 \\
\hline 88 & 57.12 & {$[\mathrm{M}-\mathrm{H}]^{-}$} & 285.0403 & $\begin{array}{c}239.0356,187.0403, \\
151.004\end{array}$ & $\mathrm{C}_{15} \mathrm{H}_{10} \mathrm{O}_{6}$ & kamepferol & -0.6 \\
\hline 89 & 57.32 & {$[\mathrm{M}-\mathrm{H}]^{-}$} & 345.0618 & $\begin{array}{l}330.0385,315.0153, \\
259.0252,187.0394\end{array}$ & $\mathrm{C}_{17} \mathrm{H}_{14} \mathrm{O}_{8}$ & syringetin & 0.6 \\
\hline 90 & 57.39 & {$[\mathrm{M}]^{+}$} & 317.0656 & $\begin{array}{l}229.0498,153.0188 \\
300.0270,271.0241\end{array}$ & $\mathrm{C}_{16} \mathrm{H}_{13} \mathrm{O}_{7}$ & petunidin & 0.0 \\
\hline 91 & 57.41 & {$[\mathrm{M}-\mathrm{H}]^{-}$} & 315.0508 & $\begin{array}{c}255.0299,227.0241 \\
151.0036\end{array}$ & $\mathrm{C}_{16} \mathrm{H}_{12} \mathrm{O}_{7}$ & isorhamnetin & -0.6 \\
\hline 92 & 58.32 & {$[\mathrm{M}+\mathrm{H}]^{+}$} & 193.1588 & 56.9419 & $\mathrm{C}_{13} \mathrm{H}_{20} \mathrm{O}$ & ionone & 0.6 \\
\hline 93 & 60.04 & {$[\mathrm{M}+\mathrm{H}]^{+}$} & 173.1536 & 76.0221 & $\mathrm{C}_{10} \mathrm{H}_{20} \mathrm{O}_{2}$ & ethyl caprylate & 0.2 \\
\hline 94 & 60.96 & {$[\mathrm{M}+\mathrm{H}]^{+}$} & 195.1748 & $\begin{array}{c}\text { 177.1634, 107.0855, } \\
77.0387\end{array}$ & $\mathrm{C}_{13} \mathrm{H}_{22} \mathrm{O}$ & theaspirane & 2.1 \\
\hline
\end{tabular}




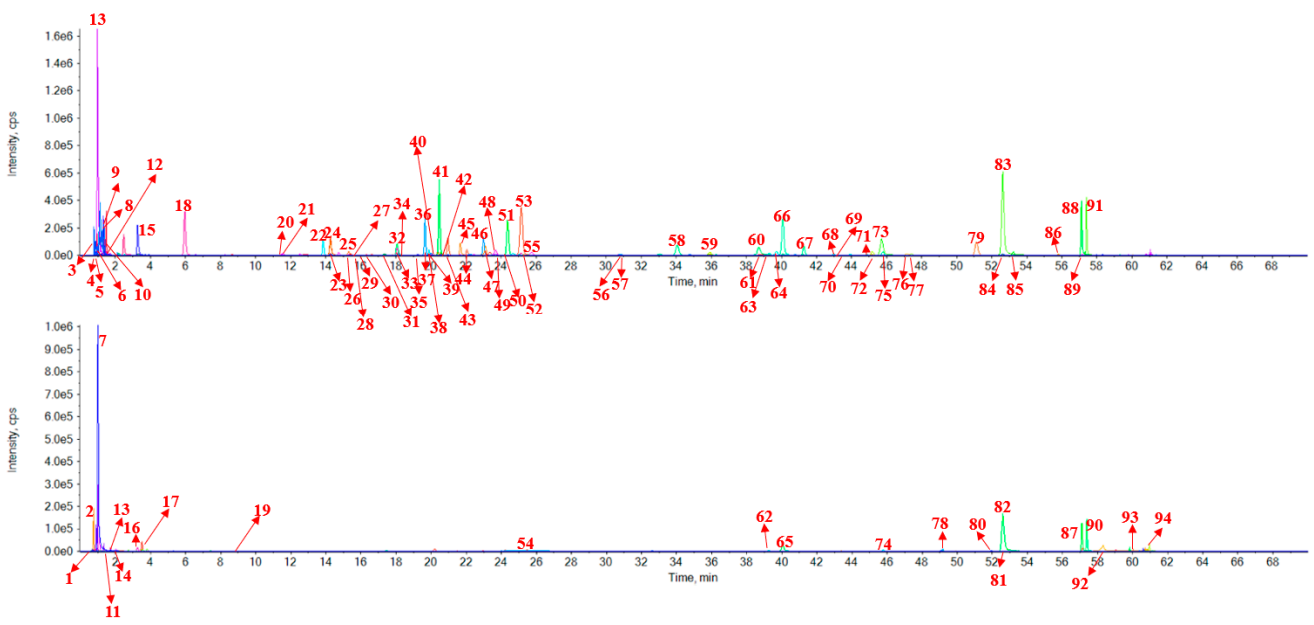

Figure 3. The extracted ion chromatogram of positive and negative ions.

\subsection{Active Ingredients of Red Wine}

The analytical data of active fractions with antioxidant activity, inhibitory activity of thrombin or lipase and 94 compounds of mass spectrometry were correlated according to retention times and peaks order. In all, 77 compounds were screened for the prevention and treatment of CHD. The corresponding relationship between fraction number and peak number of the potential active components was shown in Table 3. These active components were concentrated in phenolic acids and flavonoids. Phenolic acids were retained during 20-30 min, which had higher antioxidant and inhibitory activity against thrombin and lipase. Flavonoids were retained during 42-60 min, which had stronger antioxidant and inhibitory activity against lipase. Besides, five ensemble approaches were considered to screen the quantitative composition. The ingredients had multi-activity at the same time; the compounds had a strong single activity; the reference substance was easy to obtain; the activity of this ingredient has been reported many times in literature; the compound was abundant, respectively. The five ensemble approaches were applicated for selecting active ingredients to the quality evaluation of red wine. 63 compounds had multiple activities; 13 compounds had a strong single activity, which were protocatechuic acid, $(+)$-catechin gallate, $(+)$-epigallocatechin, acetylsalicylic acid, caftaric acid, chlorogenic acid, benzoic acid, procyanidin B7, trans-piceid, astragalin, cis-piceid, ethyl caffeate and malvidin-3-O-(6-O-coumaroylglucoside). According to the reference substance was easy to obtain and the activity of this ingredient has been reported many times in literature, a total of 19 compounds were screened, which were succinic acid, gallic acid, coumalic acid, procyanidin B1, (-)-epigallocatechin, vanillic acid, catechin, caffeic acid, syringic acid, epicatechin, p-coumaric acid, quercetin-3-O-glucuronide, isoquercitrin, isorhamnetin3-O-glucoside, trans-resveratrol, quercetin, protocatechuic acid, $(+)$-catechin gallate and (+)-epigallocatechin. Finally, based on the content of the compound from red wine, 12 compounds were screened for further determination, which were gallic acid, coumalic acid, proanthocyanidin B1, catechin, caffeic acid, isoquercitrin, protocatechin, syringic acid, epicatechin, p-coumaric acid, isorhamnetin-3-O-glucoside and quercetin. 
Table 3. The corresponding relationship between fraction number and peak number of the 77 potential active components.

\begin{tabular}{|c|c|c|c|c|c|}
\hline $\begin{array}{l}\text { Fraction } \\
\text { Number }\end{array}$ & Peak Number & Identification & $\begin{array}{l}\text { Antioxidant } \\
\text { Activity }\end{array}$ & $\begin{array}{c}\text { Thrombin } \\
\text { Inhibitory Activity }\end{array}$ & $\begin{array}{l}\text { Lipase Inhibitory } \\
\text { Activity }\end{array}$ \\
\hline $\mathrm{F} 1$ & $5,6,10,12$ & $\begin{array}{c}\text { citric acid, tartaric acid, } \\
\text { L-malic acid, shikimic acid }\end{array}$ & yes & yes & yes \\
\hline $\mathrm{F} 2$ & 12 & shikimic acid & & yes & yes \\
\hline F3 & 15,16 & $\begin{array}{l}\text { succinic acid, } \\
\text { trans-4-hydroxycinnamic acid }\end{array}$ & yes & yes & \\
\hline $\mathrm{F} 4$ & 17 & tyramine & yes & yes & \\
\hline F5 & 18 & gallic acid & yes & yes & \\
\hline F6 & 18 & gallic acid & yes & yes & \\
\hline F9 & 19 & coumalic acid & yes & yes & \\
\hline F10 & 21 & protocatechuic acid & yes & & \\
\hline F11 & 21 & protocatechuic acid & & & yes \\
\hline F13 & 22 & (+)-gallocatechin & yes & & \\
\hline F14 & 23,24 & $\begin{array}{l}\text { acetylsalicylic acid, caftaric } \\
\text { acid }\end{array}$ & & yes & \\
\hline F16 & $25,26,27,28$ & $\begin{array}{c}\text { gentisic acid, } \\
\text { protocatechualdehyde, } \\
\text { homovanillic acid, methyl } \\
\text { gallate }\end{array}$ & yes & & yes \\
\hline F17 & 29 & 4-hydroxybenzoic acid & yes & & yes \\
\hline F18 & 30 & chlorogenic acid & yes & & \\
\hline F19 & 31 & p-coutaric acid & yes & & yes \\
\hline F20 & $32,33,34$ & $\begin{array}{l}\text { m-coumaric acid, coutaric } \\
\text { acid, dimethyl succinate }\end{array}$ & & & yes \\
\hline $\mathrm{F} 21$ & 35 & $\begin{array}{l}\text { benzoic acid } \\
\text { procyanidin } \mathrm{B} 1\end{array}$ & & & yes \\
\hline $\mathrm{F} 22$ & $36,37,38$ & $\begin{array}{l}\text { (-)-epigallocatechin, } \\
\text { procyanidin B3 }\end{array}$ & yes & yes & yes \\
\hline $\mathrm{F} 23$ & $\begin{array}{c}39,40,41,42 \\
43\end{array}$ & $\begin{array}{c}\text { vanillic acid, } \\
\text { 2-Hydroxycinnamic acid, } \\
\text { catechin, fertaric acid, caffeic } \\
\text { acid }\end{array}$ & yes & & yes \\
\hline F24 & 44 & epicatechin gallate & yes & & yes \\
\hline $\mathrm{F} 25$ & 45 & syringic acid & yes & yes & yes \\
\hline F26 & $46,47,48$ & $\begin{array}{c}\text { procyanidin } \mathrm{B} 2, \mathrm{p} \text {-coumaric } \\
\text { acid glucoside, diethyl malate } \\
\text { desaminotyrosine, }\end{array}$ & yes & yes & yes \\
\hline $\mathrm{F} 27$ & $49,50,51$ & $\begin{array}{c}\text { 2-hydroxybenzoic acid, } \\
\text { epicatechin }\end{array}$ & yes & yes & yes \\
\hline F28 & 53,54 & $\begin{array}{c}\text { ethyl gallate, } \\
\text { malvidin-3-O-glucoside }\end{array}$ & yes & yes & yes \\
\hline F29 & 55 & p-coumaric acid & yes & yes & yes \\
\hline F30 & 55 & p-coumaric acid & yes & yes & yes \\
\hline F31 & 56 & homogentisic acid & yes & yes & \\
\hline F32 & 57 & procyanidin B7 & yes & & \\
\hline F36 & 58 & myricetin-3-O-galactoside & yes & yes & \\
\hline F37 & 59 & trans-piceid & yes & & \\
\hline F39 & 60,61 & $\begin{array}{c}\text { ellagic acid, dihydrocaffeic } \\
\text { acid }\end{array}$ & yes & & \\
\hline $\mathrm{F} 40$ & 62,63 & $\begin{array}{l}\text { delphinidin-3-O-glucoside, } \\
\text { hyperoside } \\
\text { delphinidin-3,5-O- }\end{array}$ & yes & & \\
\hline $\mathrm{F} 42$ & 65,66 & $\begin{array}{l}\text { diglucoside, } \\
\text { quercetin-3-O-glucuronide }\end{array}$ & yes & & yes \\
\hline
\end{tabular}


Table 3. Cont.

\begin{tabular}{|c|c|c|c|c|c|}
\hline $\begin{array}{l}\text { Fraction } \\
\text { Number }\end{array}$ & Peak Number & Identification & $\begin{array}{l}\text { Antioxidant } \\
\text { Activity }\end{array}$ & $\begin{array}{c}\text { Thrombin } \\
\text { Inhibitory Activity }\end{array}$ & $\begin{array}{c}\text { Lipase Inhibitory } \\
\text { Activity }\end{array}$ \\
\hline F43 & $67,68,69,70$ & $\begin{array}{c}\text { laricitrin-3-O-glucoside, } \\
\text { luteolin -3-O-glucoside, } \\
\text { astilbin, isoquercitrin }\end{array}$ & yes & & yes \\
\hline F44 & 70 & isoquercitrin & yes & & yes \\
\hline F45 & 71 & astragalin & & yes & \\
\hline F46 & 72 & cis-piceid & yes & & \\
\hline $\mathrm{F} 48$ & $73,74,75$ & $\begin{array}{l}\text { myricetin, } \\
\text { petunidin-3-O-glucoside, } \\
\text { isorhamnetin-3-O-glucoside }\end{array}$ & & & yes \\
\hline F49 & 76,77 & trans-resveratrol, phloridzin & & & yes \\
\hline F51 & 778 & $\begin{array}{l}\text { delphindin-3-O-(6-O- } \\
\text { acetylglucoside) }\end{array}$ & & yes & \\
\hline F54 & 79,80 & $\begin{array}{l}\text { ethyl caffeate, malvidin-3-O- } \\
\text { (6-O-coumaroylglucoside) }\end{array}$ & yes & & \\
\hline F55 & $81,82,83,84$ & $\begin{array}{l}\text { morin, delphinidin, quercetin, } \\
\text { resveratrol }\end{array}$ & yes & & yes \\
\hline F56 & 85 & laricitrin & yes & yes & yes \\
\hline F57 & 85 & laricitrin & yes & yes & yes \\
\hline F58 & 86 & naringenin & & yes & yes \\
\hline F59 & 87 & cyanidin & yes & & yes \\
\hline $\mathrm{F} 60$ & $88,89,90$ & $\begin{array}{l}\text { kamepferol, syringetin, } \\
\text { petunidin }\end{array}$ & yes & & yes \\
\hline
\end{tabular}

\subsection{UHPLC Analysis}

The UHPLC conditions were considered to obtain better chromatographic separation. The different concentrations of acid $(0.03 \%, 0.05 \%$ and $0.1 \%$ trifluoroacetic acid, $0.05 \%$ formic acid, $0.05 \%$ acetic acid and pure water), column temperatures $\left(25,30\right.$ and $\left.35{ }^{\circ} \mathrm{C}\right)$ and detection wavelength $(210,220,254$ and $280 \mathrm{~nm})$ were optimized. By comparing resolutions and the peak shapes, the better separation was achieved when $0.1 \%$ trifluoroacetic acid was selected as mobile phase, the column temperature and flow rate were optimal at $30^{\circ} \mathrm{C}$ and $0.3 \mathrm{~mL} \mathrm{~min}{ }^{-1}$, respectively. The UHPLC chromatogram was shown in Figure 4.

\subsection{Method Validation}

The precision, linearity, stability, accuracy, LODs and LOQs were validated. The RSD values of intra-day and inter-day precision were all less than $2.2 \%$ and $4.8 \%$, respectively. Overall, 12 components were stable within $24 \mathrm{~h}$ at room temperature, and RSDs were less than $2.9 \%$. The calibration curves of the 12 compounds were of high correlation coefficient $(\mathrm{r}>0.999)$ under the concentration ranges. The LODs were ranged from 0.04 to $0.75 \mu \mathrm{g} / \mathrm{mL}$ and LOQs were ranged from 0.12 to $2.0 \mu \mathrm{g} / \mathrm{mL}$ for the 12 compounds, respectively. The results were shown in (Table 4). As can be seen from Table 5, the recoveries of the 12 compounds were in the range of $83.4-106 \%$ and RSDs values were not more than $4.3 \%$. All these values were found in an acceptable range, indicating that the method was accurate, reproducible and reliable for quantification of bioactive compounds from red wine. 


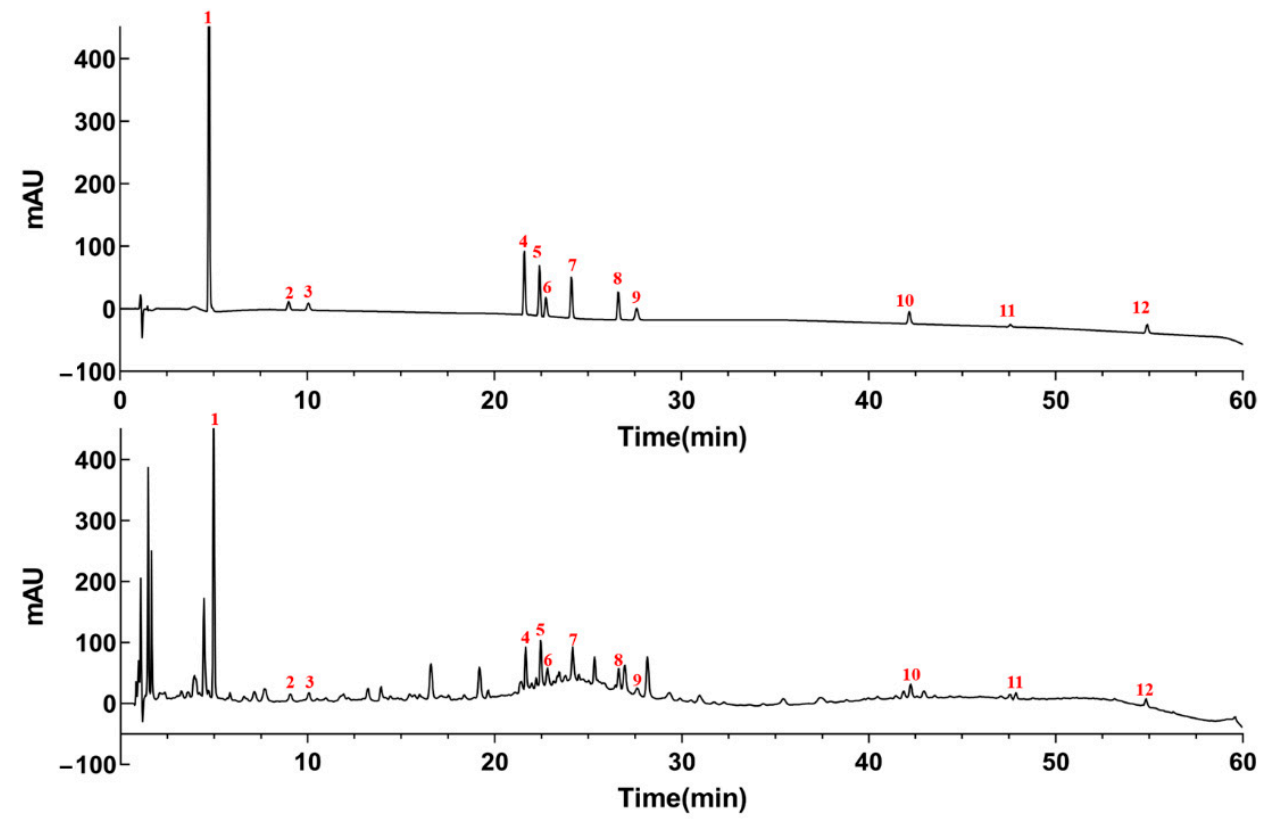

Figure 4. The typical reference chromatogram (top) and the typical chromatogram of red wine (bottom). gallic acid (1), coumalic acid (2), protocatechuic acid (3), proanthocyanidin B1 (4), catechins (5), caffeic acid (6), syringic acid (7), epicatechin (8), p-coumaric acid (9), isoquercitrin (10), isorhamnetin 3-O-glucoside (11) and quercetin (12).

Table 4. Precision, stability, linearity, LODs and LOQs of 12 compounds from red wine.

\begin{tabular}{|c|c|c|c|c|c|c|c|c|c|c|}
\hline \multirow{3}{*}{ Analytes } & \multicolumn{4}{|c|}{ Precision } & \multirow{3}{*}{$\begin{array}{l}\text { Stability } \\
\begin{array}{c}\text { RSD (\%) } \\
(n=6)\end{array}\end{array}$} & \multicolumn{3}{|c|}{ Linearity } & \multirow{3}{*}{$\underset{(\mu \mathrm{g} / \mathrm{mL})}{\mathrm{LOQ}}$} & \multirow{3}{*}{$\begin{array}{c}\text { LOD } \\
(\mu \mathrm{g} / \mathrm{mL})\end{array}$} \\
\hline & \multicolumn{3}{|c|}{$\begin{array}{c}\text { Intra-Day, RSD (\%), } \\
(n=3)\end{array}$} & \multirow{2}{*}{$\begin{array}{c}\text { Inter-Day } \\
\text { RSD (\%) } \\
(n=9)\end{array}$} & & \multirow{2}{*}{$\begin{array}{l}\text { Range } \\
(\mu \mathrm{g} / \mathrm{mL})\end{array}$} & \multirow{2}{*}{ Equation } & \multirow{2}{*}{$\mathbf{r}^{2}$} & & \\
\hline & Low & Middle & High & & & & & & & \\
\hline gallic acid & 2.2 & 2.2 & 1.3 & 3.4 & 2.5 & $20.00 \sim 320.0$ & $y=0.3627 x+0.0831$ & 0.9998 & 0.12 & 0.040 \\
\hline coumalic acid & 3.2 & 1.5 & 1.9 & 4.4 & 1.8 & $6.030 \sim 96.48$ & $y=0.2339 x-0.0078$ & 0.9994 & 2.0 & 0.75 \\
\hline $\begin{array}{l}\text { protocatechuic } \\
\text { acid }\end{array}$ & 4.9 & 2.2 & 1.7 & 4.2 & 1.1 & $1.005 \sim 16.08$ & $y=0.1498 x+0.0290$ & 0.9993 & 0.12 & 0.040 \\
\hline $\begin{array}{l}\text { proanthocyanidin } \\
\text { B1 }\end{array}$ & 3.6 & 2.1 & 1.8 & 4.6 & 2.2 & $6.000 \sim 96.0$ & $y=0.1156 x+0.0121$ & 0.9996 & 0.50 & 0.12 \\
\hline catechin & 5.0 & 1.3 & 2.0 & 3.3 & 2.5 & $6.030 \sim 96.5$ & $y=0.2149 x+0.0288$ & 0.9997 & 0.12 & 0.040 \\
\hline caffeic acid & 2.5 & 2.3 & 1.3 & 4.7 & 2.2 & $3.000 \sim 48.00$ & $y=0.2645 x+0.0428$ & 0.9992 & 0.12 & 0.040 \\
\hline syringic acid & 4.8 & 2.4 & 2.1 & 4.8 & 1.8 & $2.973 \sim 47.56$ & $y=0.5234 x+0.0300$ & 0.9997 & 0.25 & 0.08 \\
\hline epicatechin & 4.2 & 2.0 & 1.2 & 4.4 & 2.9 & $3.030 \sim 48.48$ & $y=0.3627 x+0.0831$ & 0.9998 & 0.50 & 0.12 \\
\hline p-coumaric acid & 4.4 & 1.7 & 1.4 & 4.1 & 2.1 & $3.015 \sim 48.24$ & $y=0.2339 x+0.0078$ & 0.9994 & 0.25 & 0.08 \\
\hline isoquercitrin & 4.6 & 1.9 & 1.3 & 4.6 & 1.8 & $0.2500 \sim 64.64$ & $y=0.1495 x+0.0431$ & 0.9994 & 0.25 & 0.08 \\
\hline $\begin{array}{l}\text { isorhamnetin-3- } \\
\text { O-glucoside }\end{array}$ & 5.0 & 2.1 & 1.7 & 3.3 & 2.3 & $0.990 \sim 15.84$ & $y=0.1156 x+0.0121$ & 0.9996 & 0.50 & 0.12 \\
\hline quercetin & 4.3 & 2.6 & 2.2 & 4.6 & 2.5 & $0.7500 \sim 31.46$ & $y=0.2166 x-0.0094$ & 0.9995 & 0.75 & 0.25 \\
\hline
\end{tabular}


Table 5. The recovery of 12 compounds from red wine $(n=3)$.

\begin{tabular}{|c|c|c|c|c|c|}
\hline Analytes & $\begin{array}{c}\text { Original } \\
\quad(\mu g)\end{array}$ & $\begin{array}{c}\text { Spiked } \\
(\mu g)\end{array}$ & $\begin{array}{c}\text { Found } \\
(\mu g)\end{array}$ & $\begin{array}{c}\text { Recovery } \\
(\%)\end{array}$ & $\begin{array}{c}\text { RSD } \\
(\%)\end{array}$ \\
\hline \multirow{4}{*}{ gallic acid } & \multirow{3}{*}{29.34} & 20.00 & 45.81 & 99.8 & 1.8 \\
\hline & & 40.00 & 68.85 & 97.0 & 1.4 \\
\hline & & 60.00 & 89.14 & 99.8 & 1.0 \\
\hline & \multirow{4}{*}{9.724} & 6.030 & 14.65 & 87.8 & 4.1 \\
\hline \multirow[t]{3}{*}{ coumalic acid } & & 12.06 & 21.19 & 95.1 & 2.2 \\
\hline & & 18.09 & 26.77 & 86.7 & 3.1 \\
\hline & & 1.005 & 3.720 & 83.4 & 4.3 \\
\hline \multirow[t]{3}{*}{ protocatechuic acid } & \multirow[t]{3}{*}{3.298} & 2.010 & 4.864 & 90.6 & 2.5 \\
\hline & & 3.015 & 6.338 & 98.4 & 1.4 \\
\hline & & 6.000 & 14.01 & 89.7 & 4.0 \\
\hline \multirow[t]{3}{*}{ proanthocyanidin B1 } & \multirow[t]{3}{*}{9.027} & 12.00 & 21.58 & 103.6 & 2.3 \\
\hline & & 18.00 & 26.27 & 94.1 & 2.1 \\
\hline & & 3.000 & 14.15 & 97.7 & 3.6 \\
\hline \multirow[t]{3}{*}{ catechin } & \multirow[t]{3}{*}{11.43} & 6.000 & 17.50 & 100.1 & 0.7 \\
\hline & & 9.000 & 20.61 & 100.0 & 0.3 \\
\hline & & 2.972 & 9.33 & 96.3 & 3.4 \\
\hline \multirow[t]{3}{*}{ caffeic acid } & \multirow[t]{3}{*}{6.539} & 5.945 & 13.14 & 105.0 & 2.1 \\
\hline & & 8.918 & 15.26 & 98.9 & 1.0 \\
\hline & & 2.973 & 8.97 & 97.7 & 3.4 \\
\hline \multirow[t]{3}{*}{ syringic acid } & \multirow[t]{3}{*}{6.204} & 5.945 & 12.08 & 97.5 & 3.2 \\
\hline & & 8.918 & 14.95 & 101.7 & 1.8 \\
\hline & & 3030 & 6.932 & 101.2 & 1.6 \\
\hline \multirow[t]{3}{*}{ epicatechin } & \multirow[t]{3}{*}{3.922} & 6.060 & 10.04 & 101.9 & 0.7 \\
\hline & & 9.090 & 13.18 & 105.9 & 1.4 \\
\hline & & 3.015 & 7.590 & 105.6 & 3.6 \\
\hline \multirow[t]{3}{*}{ p-coumaric acid } & \multirow[t]{3}{*}{4.473} & 6.030 & 10.15 & 92.5 & 1.1 \\
\hline & & 9.045 & 13.78 & 100.6 & 0.6 \\
\hline & & 4.040 & 12.75 & 98.1 & 3.2 \\
\hline \multirow[t]{3}{*}{ isoquercitrin } & \multirow[t]{3}{*}{8.802} & 8.080 & 16.75 & 98.8 & 2.1 \\
\hline & & 12.12 & 20.64 & 98.8 & 1.9 \\
\hline & & 0.9900 & 4.053 & 98.1 & 1.1 \\
\hline \multirow[t]{3}{*}{ isorhamnetin-3-O-glucoside } & \multirow[t]{3}{*}{3.144} & 1.980 & 5.123 & 97.4 & 2.6 \\
\hline & & 2.970 & 6.019 & 102.1 & 2.4 \\
\hline & & 1.966 & 4.928 & 95.5 & 1.6 \\
\hline \multirow[t]{2}{*}{ quercetin } & \multirow[t]{2}{*}{3.146} & 3.933 & 6.950 & 96.7 & 1.3 \\
\hline & & 5.899 & 8.82 & 92.2 & 4.1 \\
\hline
\end{tabular}

\subsection{Contents of the Bioactive Components from Red Wine}

UHPLC was applied to analyze the bioactive components in triplicates from red wine. The results showed that among the bioactive compounds, gallic acid was in the highest amounts of $46.94 \mu \mathrm{g} / \mathrm{mL}$. The contents of coumalic acid $(18.93 \mu \mathrm{g} / \mathrm{mL})$, proanthocyanidin B1 $(14.19 \mu \mathrm{g} / \mathrm{mL})$, catechin $(19.37 \mu \mathrm{g} / \mathrm{mL})$, caffeic acid $(12.05 \mu \mathrm{g} / \mathrm{mL})$ and isoquercitrin $(10.21 \mu \mathrm{g} / \mathrm{mL})$ were in the range of $10.21-19.37 \mu \mathrm{g} / \mathrm{mL}$. Protocatechin $(4.768 \mu \mathrm{g} / \mathrm{mL})$, syringic acid $(9.87 \mu \mathrm{g} / \mathrm{mL})$, epicatechin $(7.148 \mu \mathrm{g} / \mathrm{mL})$, p-coumaric acid $(8.18 \mu \mathrm{g} / \mathrm{mL})$, isorhamnetin-3-O-glucoside $(6.460 \mu \mathrm{g} / \mathrm{mL})$ and quercetin $(5.353 \mu \mathrm{g} / \mathrm{mL})$ were in low contents. Contents of 35 batches red wine were shown in (Figure 5). These components may be considered to be the main bioactive compounds from red wine and had the antioxidant activity, inhibitory activities of and lipase. 


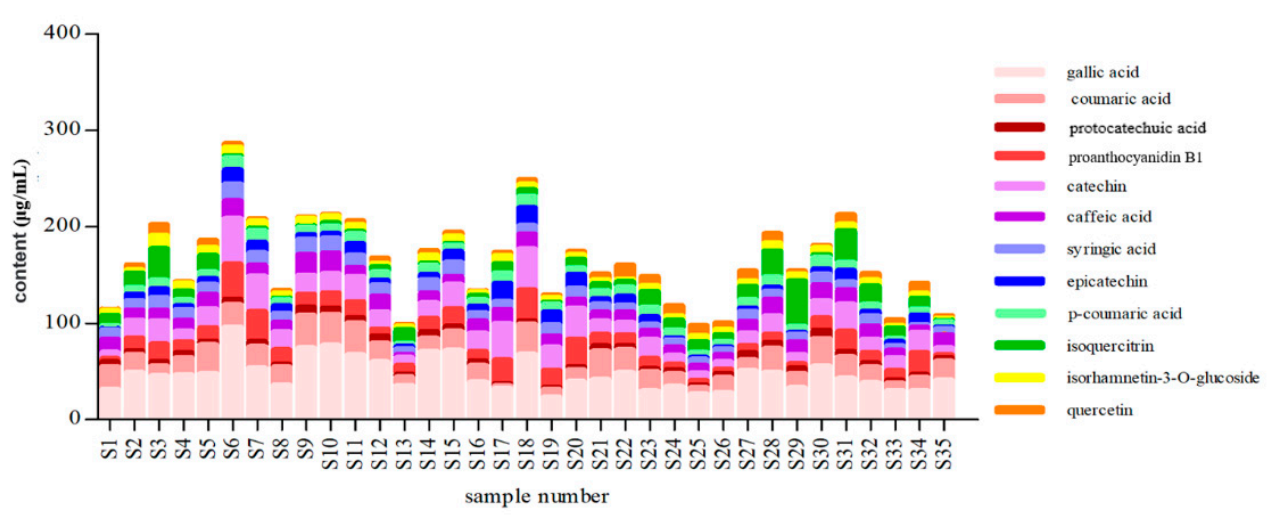

Figure 5. The content of 12 compounds from red wine $(n=3)$.

\subsection{Confirmation the Activity of the 12 Compounds}

In order to verify that the compounds in the obtained fractions, 12 active compounds mixture were contrasted for red wine in terms of antioxidant activity, thrombin inhibitory activity and lipase inhibitory activity. The results indicated that inhibition of selected 12 compounds had reached almost $90 \%$ of red wine. The inhibition ratios of 12 compounds mixture and red wine were displayed in Figure 6. Moreover, the difference between inhibition of red wine and 12 compounds mixture were also shown in Figure 6. Comparing the inhibition ratio of red wine and 12 compounds which concentrations were the same as red wine, it was shown that the inhibition ratios of 12 compounds were similar to red wine, which was verified that the screening 12 compounds could reflect the total activity of red wine in terms of antioxidant activity, thrombin inhibitory activity and lipase inhibitory activity. Accordingly, the blindness of selecting quantitative component was avoided, which could further provide reference for screening and quantification the active ingredients from nutraceutical and traditional Chinese medicine.
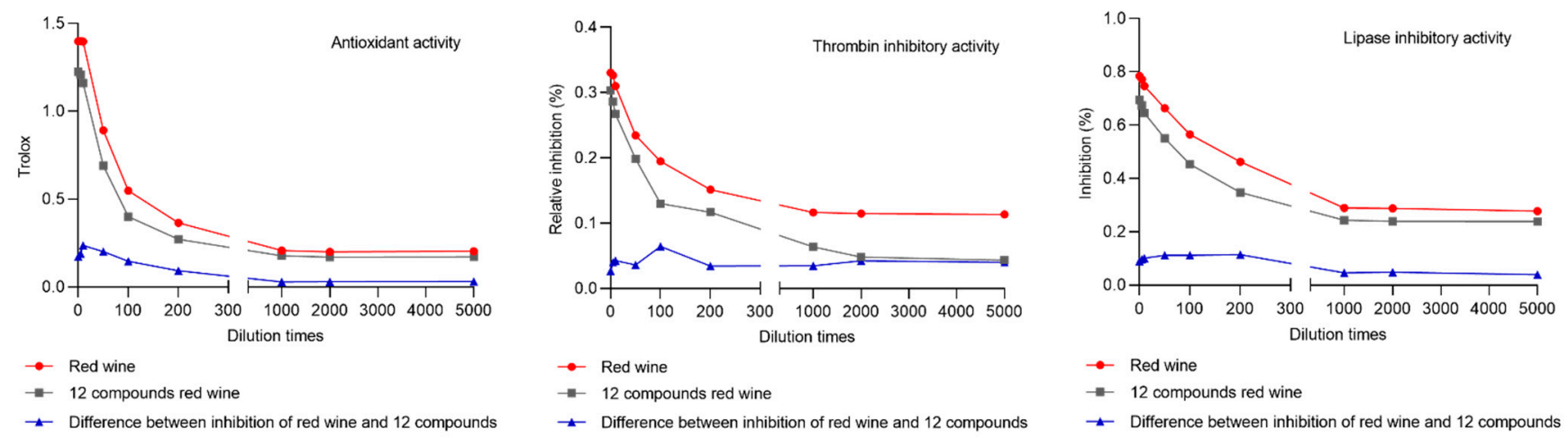

Figure 6. The inhibition ratios of red wine and 12 active compounds mixture with different dilution times and the difference between inhibition of red wine and 12 compounds mixture.

\subsection{The Application of Multi-Activity Integrated Strategy}

Red wine contained many ingredients, which had different activities such as antioxidant, anticoagulant and lipid-lowering. CHD was the results of the interaction of many complex factors. Hence, the multi-activity of red wine was corresponding to the multiple pathogenesis of CHD. Indeed, the multi-activity integrated strategy of red wine was successfully established to quality evaluation of red wine based on the screened 12 bioactive compounds to prevent CHD. Additionally, the multi-activity integrated strategy may help to discover bioactive components rapidly and efficiently, which provided reference for exploring active ingredients in food. 


\section{Materials and Methods}

\subsection{Reagents and Chemical}

HPLC-grade Acetonitrile (ACN) and methanol (MeOH) were purchased from Merck (Darmstadt, Germany). Trifluoroacetic acid (TFA) was obtained from Guangzhou Chemical Reagent Factory (Guangzhou, China). Reference Standards including gallic acid $(98.1 \%)$, catechin $(99.2 \%)$, caffeic acid $(99.7 \%)$, epicatechin $(99.7 \%)$, isoquercitrin $(98.0 \%)$ and quercetin $(98.0 \%)$ were provided from National Institutes for Food and Drug Control (Beijing, China). Coumalic acid (98.0\%), proanthocyanidin B1 (98.0\%) and syringic acid (98.0\%) were purchased from Chengdu Chroma-Biotechnology Co., Ltd. (Sichuan, China). P-coumaric acid (98.0\%) and isorhamnetin-3-O-glucoside (98.0\%) were offered by Shanghai Tauto Biotech Co., Ltd. (Shanghai, China). The water used in this study was purified by a Milli-Q water purification system (MA, USA). Total Antioxidant Capacity Assay Kit (ABTS) was supplied from Beyotime (Shanghai, China). Thrombin Inhibitor Screening Kit (Fluorometric) was obtained from Biovision (San Francisco, CA, USA). Lipase (TypeII, L3126) was purchased from Solarbio Science Co. Ltd. (Beijing, China) and 4-Methylumbelliferyl Oleate (4-MUO) was provided by Sigma-Aldrich (St. Louis, MO, USA). Red wine (2018 vintage, the central valley of Chile, alcoholic degree $13.0 \%(v / v)$ ) was made of cabernet sauvignon, which was obtained from Ole' Boutique Supermarket (Shenzhen, Guangdong, China).

\subsection{Sample and Standard Solution Preparation}

The red wine was analyzed immediately after the bottle opening. The sample $(2 \mathrm{~mL})$ was filtrated through a $0.22 \mu \mathrm{m}$ polyvinylidene fluoride membrane. Appropriate amounts of gallic acid, catechin, caffeic acid, epicatechin, isoquercitrin, coumalic acid, proanthocyanidin B1, syringic acid, p-coumaric acid and isorhamnetin-3-O-glucoside were precisely weighed and dissolved with methanol to prepare standards solutions of $1 \mathrm{mg} / \mathrm{mL}$. All the samples and standard solutions were stored at $4{ }^{\circ} \mathrm{C}$ in the dark for analysis.

\subsection{Preparation of the Red Wine Fractions}

Fraction collector (BSZ-100, Shanghai Jiapeng Technology Instrument, Shanghai, China) was used to prepare fractions. The red wine sample was injected into the $\mathrm{UH}-$ PLC system for separation. Then fractions were collected every $60 \mathrm{~s}$ by setting the fraction collector and evaporated to dryness by nitrogen gas. The residues were dissolved by methyl alcohol:water (1:1).

\subsection{UHPLC Analysis}

The UHPLC analysis was performed using an Dionex ultra high performance liquid chromatograph (Dionex UltiMate 3000, Thermo, MA, USA) equipped with a Dionex UltiMate 3000 pump, a Dionex UltiMate 3000 autosampler and a Dionex UltiMate 3000 diode array detector. The chromatographic separation was achieved on an ACQUITY UPLC column (HSS T3, $1.8 \mu \mathrm{m}, 2.1 \mathrm{~mm} \times 100 \mathrm{~mm}$, Waters, Ireland) [49] at $30^{\circ} \mathrm{C}$. The mobile phase was composed of water containing $0.1 \%$ TFA (A) [50] and methanol (B) at a flow rate of $0.3 \mathrm{~mL} / \mathrm{min}$. The elution program was conducted as follows: $0-15 \mathrm{~min}$ at $0-6 \% \mathrm{~B} ; 15-20 \mathrm{~min}$ at $6-11 \% \mathrm{~B} ; 20-30 \mathrm{~min}$ at $11-11 \% \mathrm{~B} ; 30-45 \mathrm{~min}$ at $11-20 \% \mathrm{~B} ; 45-55 \mathrm{~min}$ at $20-30 \% \mathrm{~B} ; 55-60 \mathrm{~min}$ at $30-90 \% \mathrm{~B}$. The injection volume was $2 \mu \mathrm{L}$. DAD wavelength was set as $220 \mathrm{~nm}$. Method validation was accorded to the Chinese Pharmacopeia guidelines, which were included linearity, limits of detection (LOD), limits of quantification (LOQ), repeatability, precision, stability and recovery.

\subsection{UFLC/Q-TOF-MS Analysis}

The identification was performed on a hybrid quadrupole time-of-flight tandem mass spectrometry (Exion-LC ${ }^{\mathrm{TM}}$ / X500R QTOF, AB SCIEX, Foster City, CA) equipped with an electrospray ionization (ESI) interface. The mass spectrometer was operated both in positive and negative ion mode. The parameters were set as following: the ion spray voltage of $5500 /-4500 \mathrm{~V}$; turbo spray temperature (TEM) of $550{ }^{\circ} \mathrm{C}$; declustering potential 
(DP) of $\pm 70 \mathrm{~V}$; collision energy (CE) of $\pm 40 \mathrm{~V}$; nebulizer gas (gas 1) of $50 \mathrm{psi}$; heater gas (gas 2) of 50 psi and curtain gas of 30 psi [20,43]. Nitrogen was kept as the nebulizer and auxiliary gas. TOF/MS and TOF/MS-MS were scanned with the mass range of $\mathrm{m} / \mathrm{z}$ 100-1200 and 50-1200, respectively. The experiments were run with $200 \mathrm{~ms}$ accumulation time for TOF/MS and $80 \mathrm{~ms}$ accumulation time for TOF/MS-MS. Continuous recalibration was carrying out at each $3 \mathrm{~h}$. In addition, dynamic background subtraction (DBS) trigger information-dependent acquisition (IDA) was used to trigger acquisition of MS/MS of low-level constituents. The accurate mass and composition for the precursor ions and fragment ions were analyzed using the SCIEX OS software integrated with the instrument.

\subsection{Antioxidant Activity Assay}

After collection fractions, the antioxidant activity was tested for the 60 fractions by Total Antioxidant Capacity Assay Kit (ABTS). ABTS was oxidized to ABTS cation radical $(\mathrm{ABTS} \bullet+)$ with appropriate oxidizing agent. The red wine has inhibited the production of ABTS - that can be detected by the absorbance at $734 \mathrm{~nm}$. Initially, $0.4 \mathrm{~mL}$ ABTS stock solution was mixed with $0.4 \mathrm{~mL}$ of the oxidizing agent solution for the preparation of the radical. This solution was maintained at room temperature for 12-16 $\mathrm{h}$ in an amber bottle. Subsequently, the mixture was diluted in PBS to obtain an absorbance of approximately $0.7 \pm 0.05$. Finally, $200 \mu \mathrm{M}$ ABTS was mixed with different fraction. The absorbance change at $734 \mathrm{~nm}$ was measured after $5 \mathrm{~min}$. The reaction solution with PBS instead of test sample was used as a control test. The total antioxidant activity was expressed as Trolox-equivalent Antioxidant Capacity (TEAC) equivalent [51,52].

\subsection{Thrombin Inhibitory Activity Assay}

The inhibitory activity against thrombin was assessed by Thrombin Inhibitor Screening Kit (Fluorometric). Thrombin inhibitor screening principle was utilized the ability of thrombin to cleave a synthetic AMC-based peptide substrate to release AMC, which can be detected by measuring fluorescence intensity at $E x / E m=350 / 450 \mathrm{~nm}$. In the presence of thrombin inhibitors, the extent of cleavage reaction was reduced or completely abolished. The loss in fluorescence intensity can be correlated to the amount of inhibitor present in the assay solution. At first, the thrombin enzyme solution was prepared as $48 \mu \mathrm{L}$ thrombin assay buffer and $2 \mu \mathrm{L}$ thrombin enzyme stock solution in each well. Then, fractions were dissolved by methyl alcohol:water (1:1) and diluted to 10 times with thrombin assay buffer. The $10 \mu \mathrm{L}$ diluted fractions or thrombin inhibitor control were added into the thrombin enzyme containing wells. The thrombin inhibitor control was consisted of $1 \mu \mathrm{L}$ thrombin inhibitor and $9 \mu \mathrm{L}$ thrombin assay buffer to thrombin enzyme well. The sample was incubated at room temperature for $15 \mathrm{~min}$. Finally, the thrombin substrate solution was added into each well, which was consisted of $35 \mu \mathrm{L}$ thrombin assay buffer and $5 \mu \mathrm{L}$ thrombin substrate. Fluorescence was measured in a kinetic mode for $30-60 \mathrm{~min}$ at $37^{\circ} \mathrm{C}$ $(E x / E m=350 / 450 \mathrm{~nm})$. The time points $\left(T_{1}=5 \mathrm{~min}, \mathrm{~T}_{2}=10 \mathrm{~min}\right)$ were chosen in the linear range of the plot and obtain the corresponding values for the fluorescence (RFU1, RFU2). Irreversible inhibitors that inhibit the thrombin activity completely at the tested concentration will have $\triangle \mathrm{RFU}=0$ and will show $100 \%$ relative inhibition. The inhibition (\%) was calculated by (slope of enzyme control - slope of fraction)/slope of enzyme control $\times 100 \%[53]$.

\subsection{Lipase Inhibitory Activity Assay}

In this study, the inhibition of each fraction was assayed by measuring the fluorescence intensity of 4-methylumbelliferyl oleate (4-MU) (the hydrolytic product of 4-MUO). The assay was performed according to the method described with slight modifications. Initially, the concentrations of trypsin $(0.83-30,000 \mathrm{U} / \mathrm{mL})$ and time were investigated to establish linear range with the concentration of 4-MUO at $0.1 \mathrm{mM}$. Then, $25 \mu \mathrm{L}$ fractions and $25 \mu \mathrm{L}$ lipase solutions were mixed together and $50 \mu \mathrm{L} 4-\mathrm{MUO}$ was added to the mixture. Finally, the amount of 4-MU was measured by microplate reader at an excitation wavelength of 
$355 \mathrm{~nm}$ and an emission wavelength of $460 \mathrm{~nm}$ after incubating at $25^{\circ} \mathrm{C}$ for $30 \mathrm{~min}$. The inhibition of pancreatic lipase activity was calculated as follows: inhibition $(\%)=\left[\left(\mathrm{A}_{\text {control }}\right.\right.$ $\left.\left.-A_{\text {sample }}\right) / A_{\text {control }}-\left(A_{\text {control }}-A_{\text {control sample }}\right) / A_{\text {control }}\right] \times 100 \%$, where $A_{\text {sample was the }}$ fluorescence of the reactions with added fraction, $A_{\text {control }}$ was the control, and $A_{\text {control sample }}$ was the fluorescence of the solvent of the fraction [12,54].

\subsection{Statistical Analyses}

The results of precision and recovery have been measured in triplicates and the stability have been measured in sextuplicate, which were expressed as mean and RSD. The content of 12 compounds have been measured in triplicates and expressed as mean.

\section{Conclusions}

In this study, the multi-activity integrated strategy was proposed to screen, identify and quantify components from red wine for prevention of CHD via UHPLC-FC and UFLCQ-TOF/MS. The multi-activity integrated strategy of red wine was established and verified the availability of screening the preventing CHD compounds from the complex sample. Red wine exhibited potential antioxidant activity and inhibitory activity against thrombin and lipase, which could be used to prevent CHD. In addition, the system had advantages over traditional methods in screening potential active compounds. The multi-activity integrated strategy may help to discover bioactive components rapidly and efficiently, which provided reference for exploring the health care function in food.

Supplementary Materials: The following are available online: Figures S1-S94: The MS spectrums of all compounds.

Author Contributions: Conceptualization, K.B.; data collection and methodology, Y.G., X.-a.Y., J.W., G.Y. and B.W.; data analysis, Y.G. and X.-a.Y.; writing—original draft preparation, Y.G. and X.-a.Y.; writing-review and editing, T.W. and K.B. All authors have read and agreed to the published version of the manuscript.

Funding: This research was funded by National Natural Science Foundation of China (No. 82104357).

Institutional Review Board Statement: Not applicable.

Informed Consent Statement: Not applicable.

Data Availability Statement: The MS spectrums of all compounds were shown in supplementary materials (Figures S1-S94).

Conflicts of Interest: The authors declare no conflict of interest.

Sample Availability: The informations of samples were shown in Supplementary Materials (Table S1).

\section{References}

1. Rodrigo, R.; Miranda, A.; Vergara, L. Modulation of endogenous antioxidant system by wine polyphenols in human disease. Clin. Chim. Acta 2011, 412, 410-424. [CrossRef] [PubMed]

2. Castaldo, L.; Narváez, A.; Izzo, L.; Graziani, G.; Gaspari, A.; Di Minno, G.; Ritieni, A. Red wine consumption and cardiovascular health. Molecules 2019, 24, 3626. [CrossRef]

3. Renaud, S.D.; de Lorgeril, M. Wine, alcohol, platelets, and the French paradox for coronary heart disease. Lancet 1992, 339, 1523-1526. [CrossRef]

4. Snopek, L.; Mlcek, J.; Sochorova, L.; Baron, M.; Hlavacova, I.; Jurikova, T.; Sochor, J. Contribution of red wine consumption to human health protection. Molecules 2018, 23, 1684. [CrossRef] [PubMed]

5. World Health Statistics 2019: Monitoring Health for the SDGs, Sustainable Development Goals. Available online: https: / /apps.who.int/iris/handle/10665/324835 (accessed on 21 May 2019).

6. Liu, P.; Zhou, B.; Gu, D.; Zhang, L.; Han, Z. Endothelial progenitor cell therapy in atherosclerosis: A double-edged sword. Ageing Res. Rev. 2009, 8, 83-93. [CrossRef] [PubMed]

7. Ceriello, A.; Assaloni, R.; Da Ros, R.; Maier, A.; Piconi, L.; Quagliaro, L.; Esposito, K.; Giugliano, D. Effect of atorvastatin and irbesartan, alone and in combination, on postprandial endothelial dysfunction, oxidative stress, and inflammation in type 2 diabetic patients. Circulation 2005, 111, 2518-2524. [CrossRef] 
8. Pothineni, N.; Subramany, S.; Kuriakose, K.; Shirazi, L.F.; Romeo, F.; Shah, P.K.; Mehta, J.L. Infections, atherosclerosis, and coronary heart disease. Eur. Heart J. 2017, 38, 3195-3201. [CrossRef]

9. Yan, Y.L.; Qiu, B.; Hu, L.J.; Jing, X.D.; Liu, Y.J.; Deng, S.B.; Du, J.L.; She, Q. Efficacy and safety evaluation of intensive statin therapy in older patients with coronary heart disease: A systematic review and meta-analysis. Eur. J. Clin. Pharmacol. 2013, 69, 2001-2009. [CrossRef]

10. Divakaran, S.; Loscalzo, J. The role of nitroglycerin and other nitrogen oxides in cardiovascular therapeutics. J. Am. Coll. Cardiol. 2017, 70, 2393-2410. [CrossRef]

11. Demartini, C.; Greco, R.; Zanaboni, A.M.; Sances, G.; De Icco, R.; Borsook, D.; Tassorelli, C. Nitroglycerin as a comparative experimental model of migraine pain: From animal to human and back. Prog. Neurobiol. 2019, 177, 15-32. [CrossRef]

12. Chang, Y.X.; Ge, A.H.; Donnapee, S.; Li, J.; Bai, Y.; Liu, J.; Gao, X.M. The multi-targets integrated fingerprinting for screening anti-diabetic compounds from a Chinese medicine Jinqi Jiangtang Tablet. J. Ethnopharmacol. 2015, 164, 210-222. [CrossRef]

13. Huang, X.; Kong, L.; Li, X.; Chen, X.; Guo, M.; Zou, H. Strategy for analysis and screening of bioactive compounds in traditional Chinese medicines. J. Chromatogr. B 2004, 812, 71-84. [CrossRef]

14. Kang, H.J.; Yang, H.J.; Kim, M.J.; Han, E.S.; Kim, H.J.; Kwon, D.Y. Metabolomic analysis of meju during fermentation by ultra performance liquid chromatography-quadrupole-time of flight mass spectrometry (UPLC-Q-TOF MS). Food Chem. 2011, 127, 1056-1064. [CrossRef]

15. He, Q.; Huang, S.; Wu, Y.; Zhang, W.; Wang, F.; Cao, J.; Ou, W.B. Comparative study on the composition of free amino acids and derivatives in the two botanical origins of an edible Chinese herb "Xiebai", i.e., Allium chinense G. Don and Allium macrostemon Bunge species. Food Res. Int. 2018, 106, 446-457. [CrossRef]

16. Konya, Y.; Taniguchi, M.; Fukusaki, E. Novel high-throughput and widely-targeted liquid chromatography-time of flight mass spectrometry method for d-amino acids in foods. J. Biosci. Bioeng. 2017, 123, 126-133. [CrossRef]

17. Cellar, N.A.; McClure, S.C.; Salvati, L.M.; Reddy, T.M. A new sample preparation and separation combination for precise, accurate, rapid, and simultaneous determination of vitamins B1, B2, B3, B5, B6, B7, and B9 in infant formula and related nutritionals by LC-MS/MS. Anal. Chim. Acta. 2016, 934, 180-185. [CrossRef]

18. Hu, H.; Xiao, L.; Zheng, B.; Wei, X.; Ellis, A.; Liu, Y.M. Identification of chemical markers in Cordyceps sinensis by HPLC-MS/MS Anal. Bioanal Chem. 2015, 407, 8059-8066. [CrossRef] [PubMed]

19. Li, Z.; Wang, Y.; Ouyang, H.; Lu, Y.; Qiu, Y.; Feng, Y.; Yang, S. A novel dereplication strategy for the identification of two new trace compounds in the extract of Gastrodia elata using UHPLC/Q-TOF-MS/MS. J. Chromatogr. B 2015, 988, 45-52. [CrossRef] [PubMed]

20. Ramabulana, A.T.; Steenkamp, P.; Madala, N.; Dubery, I.A. Profiling of chlorogenic acids from bidens pilosa and differentiation of closely related positional isomers with the aid of UHPLC-QTOF-MS/MS-based in-source collision-induced dissociation. Metabolites 2020, 10, 178. [CrossRef]

21. Fattahi, A.; Shakeri, A.; Tayarani-Najaran, Z.; Kharbach, M.; Segers, K.; Heyden, Y.V.; Asili, J. UPLC-PDA-ESI-QTOF-MS/MS and GC-MS analysis of Iranian Dracocephalum moldavica L. Food Sci. Nutr. 2021. [CrossRef] [PubMed]

22. Dhananjeyan, M.R.; Bykowski, C.; Trendel, J.A.; Sarver, J.G.; Ando, H.; Erhardt, P.W. Simultaneous determination of procaine and para-aminobenzoic acid by LC-MS/MS method. J. Chromatogr. B 2007, 847, 224-230. [CrossRef] [PubMed]

23. Kivrak, İ. Analytical methods applied to assess chemical composition, nutritional value and in vitro bioactivities of Terfezia olbiensis and Terfezia claveryi from Turkey. Food Anal. Methods 2015, 8, 1279-1293. [CrossRef]

24. Boğa, M.; Ertaş, A.; Yılmaz, M.A.; Kızıl, M.; Çeken, B.; Haşimi, N.; Deveci, Ö. UHPLC-ESI-MS/MS and GC-MS analyses on phenolic, fatty acid and essential oil of Verbascum pinetorum with antioxidant, anticholinesterase, antimicrobial and DNA damage protection effects. Iran. J. Pharm. Res. 2016, 15, 393-405. Available online: https://www.ncbi.nlm.nih.gov/pmc/articles/PMC514 9026/ (accessed on 11 July 2021).

25. Jeszka-Skowron, M.; Zgoła-Grześkowiak, A.; Frankowski, R. Cistus incanus a promising herbal tea rich in bioactive compounds: LC-MS/MS determination of catechins, flavonols, phenolic acids and alkaloids-A comparison with Camellia sinensis, Rooibos and Hoan Ngoc herbal tea. J. Food Compost Anal. 2018, 74, 71-81. [CrossRef]

26. Gajula, R.; Pilli, N.R.; Ravi, V.B.; Maddela, R.; Inamadugu, J.K.; Polagani, S.R.; Busa, S. Simultaneous determination of atorvastatin and acetylsalicylic acid in human plasma by LC-MS/MS: Its pharmacokinetic application. Sci. Pharm. 2012, 80, 923-940. [CrossRef]

27. Hossain, M.B.; Rai, D.K.; Brunton, N.P.; Martin-Diana, A.B.; Barry-Ryan, C. Characterization of phenolic composition in Lamiaceae spices by LC-ESI-MS/MS. J. Agric. Food Chem. 2010, 58, 10576-10581. [CrossRef]

28. Kammerer, D.; Claus, A.; Carle, R.; Schieber, A. Polyphenol screening of pomace from red and white grape varieties (Vitis vinifera L.) by HPLC-DAD-MS/MS. J. Agric. Food Chem. 2004, 52, 4360-4367. [CrossRef] [PubMed]

29. Peng, J.; Guo, K.; Xia, J.; Zhou, J.; Yang, J.; Westaway, D.; Li, L. Development of Isotope labeling liquid chromatography mass spectrometry for mouse urine metabolomics: Quantitative metabolomic study of transgenic mice related to Alzheimer's disease. J. Proteome Res. 2014, 13, 4457-4469. [CrossRef] [PubMed]

30. Domínguez-Fernández, M.; Yang, P.Y.T.; Ludwig, I.A.; Clifford, M.N.; Cid, C.; Rodriguez-Mateos, A. In vivo study of the bioavailability and metabolic profile of (poly) phenols after sous-vide artichoke consumption. Food Chem. 2022, 367, 130620. [CrossRef]

31. Zhang, H.; Shao, Y.; Bao, J.; Beta, T. Phenolic compounds and antioxidant properties of breeding lines between the white and black rice. Food Chem. 2015, 172, 630-639. [CrossRef] [PubMed] 
32. Yan, L.; Yin, P.; Ma, C.; Liu, Y. Method development and validation for pharmacokinetic and tissue distributions of ellagic acid using ultrahigh performance liquid chromatography-tandem mass spectrometry (UPLC-MS/MS). Molecules 2014, 19, 18923-18935. [CrossRef] [PubMed]

33. Nuengchamnong, N.; Krittasilp, K.; Ingkaninan, K. Characterisation of phenolic antioxidants in aqueous extract of Orthosiphon grandiflorus tea by LC-ESI-MS/MS coupled to DPPH assay. Food Chem. 2011, 127, 1287-1293. [CrossRef]

34. Dall'Asta, M.; Calani, L.; Tedeschi, M.; Jechiu, L.; Brighenti, F.; Del Rio, D. Identification of microbial metabolites derived from in vitro fecal fermentation of different polyphenolic food sources. Nutrition 2012, 28, 197-203. [CrossRef]

35. Todoroki, K.; Ishii, Y.; Miyauchi, C.; Kitagawa, S.; Min, J.Z.; Inoue, K.; Toyo'oka, T. Simple and sensitive analysis of histamine and Tyramine in Japanese soy sauces and their intermediates using the stable isotope dilution HILIC-MS/MS method. J. Agric. Food Chem. 2014, 62, 6206-6211. [CrossRef] [PubMed]

36. Mazzotti, F.; Benabdelkamel, H.; Di Donna, L.; Maiuolo, L.; Napoli, A.; Sindona, G. Assay of tyrosol and hydroxytyrosol in olive oil by tandem mass spectrometry and isotope dilution method. Food Chem. 2012, 135, 1006-1010. [CrossRef]

37. Liu, Z.; Ezernieks, V.; Reddy, P.; Elkins, A.; Krill, C.; Murphy, K.; Spangenberg, G. A simple GC-MS/MS method for determination of smoke taint-related volatile phenols in grapes. Metabolites 2020, 10, 294. [CrossRef] [PubMed]

38. Schmidt, L.; Heck, N.D.V.; Ferreira, I.; Göethel, G.; Somacal, S.; Emanuelli, T.; Augusti, P.R. Ochratoxin A presence in Cabernet Sauvignon wine changes antioxidant activity in vitro and oxidative stress markers in vivo. Food Addit. Contam. Part A Chem. Anal. Control Expo. Risk Assess 2020, 37, 1755-1764. [CrossRef]

39. Arimboor, R.; Arumughan, C. HPLC-DAD-MS/MS profiling of antioxidant flavonoid glycosides in sea buckthorn (Hippophae rhamnoides L.) seeds. Int. J. Food Sci. Nutr. 2012, 63, 730-738. [CrossRef]

40. Hong, Y.; Liao, X.; Chen, Z. Determination of bioactive components in the fruits of Cercis chinensis Bunge by HPLC-MS/MS and quality evaluation by principal components and hierarchical cluster analysis. J. Pharm. Anal. 2020. [CrossRef]

41. Hubner, A.; Sobreira, F.; Vetore Neto, A.; Pinto, C.A.S.D.O.; Dario, M.F.; Díaz, I.E.C.; Bacchi, E.M. The synergistic behavior of antioxidant phenolic compounds obtained from winemaking waste's valorization, increased the efficacy of a sunscreen system. Antioxidants 2019, 8, 530. [CrossRef]

42. Zhang, Q.F.; Guo, Y.X.; Zheng, G.; Wang, W.J. Chemical constituents comparison between Rhizoma Smilacis Glabrae and Rhizoma Smilacis Chinae by HPLC-DAD-MS/MS. Nat. Prod. Res. 2013, 27, 277-281. [CrossRef] [PubMed]

43. Tetik, M.A.; Sevindik, O.; Kelebek, H.; Selli, S. Screening of key odorants and anthocyanin compounds of cv. Okuzgozu (Vitis vinifera L.) red wines with a free run and pressed pomace using GC-MS-Olfactometry and LC-MS-MS. J. Mass Spectrom. 2018, 53, 444-454. [CrossRef] [PubMed]

44. Ha, T.J.; Park, J.E.; Lee, K.S.; Seo, W.D.; Song, S.B.; Lee, M.H.; Lee, J.H. Identification of anthocyanin compositions in black seed coated Korean adzuki bean (Vigna angularis) by NMR and UPLC-Q-Orbitrap-MS/MS and screening for their antioxidant properties using different solvent systems. Food Chem. 2021, 346, 128882. [CrossRef] [PubMed]

45. Sun, J.; Liang, F.; Bin, Y.; Li, P.; Duan, C. Screening non-colored phenolics in red wines using liquid chromatography/ultraviolet and mass spectrometry/mass spectrometry libraries. Molecules 2007, 12, 679-693. [CrossRef] [PubMed]

46. Liu, J.; Mu, Y.; Xiong, S.; Sun, P.; Deng, Z. A UPLC-MS/MS method for comparative pharmacokinetics study of morusin and morin in normal and diabetic rats. Biomed. Chromatogr. 2019, 33, e4516. [CrossRef]

47. De Rosso, M.; Panighel, A.; Vedova, A.D.; Gardiman, M.; Flamini, R. Characterization of non-anthocyanic flavonoids in some hybrid red grape extracts potentially interesting for industrial uses. Molecules 2015, 20, 18095-18106. [CrossRef]

48. Ferreira, V.; López, R.; Cacho, J.F. Quantitative determination of the odorants of young red wines from different grape varieties. J. Agric. Sci. 2000, 80, 1659-1667. [CrossRef]

49. Yang, L.; Jiang, H.; Xing, X.; Yan, M.; Guo, X.; Man, W.; Yang, L. A biosensor-based quantitative analysis system of major active ingredients in Lonicera japonica thunb. Using UPLC-QDa and chemometric analysis. Molecules 2019, 24, 1787. [CrossRef]

50. Abass, K.; Reponen, P.; Mattila, S.; Pelkonen, O. Metabolism of $\alpha$-thujone in human hepatic preparations in vitro. Xenobiotica 2011, 41, 101-111. [CrossRef]

51. Thaipong, K.; Boonprakob, U.; Crosby, K.; Cisneros-Zevallos, L.; Byrne, D.H. Comparison of ABTS, DPPH, FRAP, and ORAC assays for estimating antioxidant activity from guava fruit extracts. J. Food Compost. Anal. 2006, 19, 669-675. [CrossRef]

52. Jasprica, I.; Bojic, M.; Mornar, A.; Besic, E.; Bucan, K.; Medic-Saric, M. Evaluation of antioxidative activity of croatian propolis samples using DPPH· and ABTS + stable free radical assays. Molecules 2007, 12, 1006-1021. [CrossRef] [PubMed]

53. Baburajeev, C.P.; Mohan, C.D.; Pandey, V.; Rangappa, S.; Shivalingegowda, N.; Kalash, L.; Rangappa, K.S. Synthesis of CC, CN coupled novel substituted dibutyl benzothiazepinone derivatives and evaluation of their thrombin inhibitory activity. Bioorg. Chem. 2019, 87, 142-154. [CrossRef] [PubMed]

54. Liu, P.K.; Weng, Z.M.; Ge, G.B.; Li, H.L.; Ding, L.L.; Dai, Z.R.; Hou, J. Biflavones from Ginkgo biloba as novel pancreatic lipase inhibitors: Inhibition potentials and mechanism. Int. J. Biol. Macromol. 2018, 118, 2216-2223. [CrossRef] [PubMed] 